\title{
OPTION PRICING WHERE THE UNDERLYING ASSETS FOLLOW A GRAM/CHARLIER DENSITY OF ARBITRARY ORDER
}

\author{
ERIK SCHLÖGL
}

Date. This version: August 23, 2012. The author would like to thank Martin Schweizer, John van der Hoek, Mark Capogreco and two anonymous referees for helpful comments on earlier versions of this paper - the usual disclaimers apply. This research was supported under the Australian Research Council's Linkage funding scheme (project number LP0562616). School of Finance and Economics, University of Technology, Sydney, PO Box 123, Broadway, NSW 2007, Australia. E-mail: Erik.Schlogl@uts.edu.au. 
ABSTRACT. If a probability distribution is sufficiently close to a normal distribution, its density can be approximated by a Gram/Charlier Series A expansion. In option pricing, this has been used fit risk-neutral asset price distributions to the implied volatility smile, ensuring an arbitrage-free interpolation of implied volatilities across exercise prices. However, the existing literature is restricted to truncating the series expansion after the fourth moment. This paper presents an option pricing formula in terms of the full (untruncated) series and discusses a fitting algorithm, which ensures that a series truncated at a moment of arbitrary order represents a valid probability density. While it is well known that valid densities resulting from truncated Gram/Charlier Series A expansions do not always have sufficient flexibility to fit all market-observed option prices perfectly, this paper demonstrates that option pricing in a model based on these densities is as tractable as the (far less flexible) original model of Black and Scholes (1973), allowing non-trivial higher moments such as skewness, excess kurtosis and so on to be incorporated into the pricing of exotic options: Generalising the Gram/Charlier Series A approach to the multiperiod, multivariate case, a model calibrated to standard option prices is developed, in which a large class of exotic payoffs can be priced in closed form. Furthermore, this approach, when applied to a foreign exchange option market involving several currencies, can be used to ensure that the volatility smiles for options on the cross exchange rate are constructed in a consistent, arbitrage-free manner.

JEL classification: C40; C63; G13; F31

Keywords: Hermite expansion; Semi-nonparametric estimation; Risk-neutral density; Option-implied distribution; Exotic option; Currency option

\section{INTRODUCTION}

Ever since the seminal paper of Breeden and Litzenberger (1978), it has been recognised that given liquid option prices for a continuum of strike prices and a fixed maturity, one can imply the risk-neutral density for the distribution of the future value of the underlying 
asset. These densities should not be interpreted in terms of probabilities under which the underlying asset will take on some value; rather, they represent state prices in the sense of Arrow (1964) and Debreu (1959). Each state price is the market value today of a unit payoff in a given future "state of the world". These implied distributions are very useful when pricing further contingent claims on the same underlying asset, which can be valued by simply integrating their future (contingent) payoffs with respect to the state price densities. Historical probabilities, calculated from time series data by some statistical method, do not enter the equation. Arguably, fitting a model to state price densities is the most direct way of ensuring consistency with current market prices. In market practice, this consistency is the foremost requirement placed on models for the pricing and risk management of derivative financial instruments.

From a more theoretical viewpoint, it also seems preferable to imply model parameters from liquid prices whenever possible, rather than from statistical estimation. In the work that followed Black and Scholes (1973), ${ }^{1}$ it has become clear that the drift under the historical probability measure does not matter for the arbitrage pricing of financial derivatives. For volatility parameters, it is important to note that the future volatility determines the value of an option, whereas statistical estimation is necessarily based on past observations. If one accepts the argument that the market efficiently aggregates all relevant information and communicates it via prices, volatility as implied from option prices is the best estimate of the future volatility under the probability measure used for pricing derivatives. Note that this is not contradicted by the apparent empirical observation that implied volatility is, at best, a biased estimate of future realised volatility. ${ }^{2}$ For one, Penttinen (2001) finds that this bias can be explained, in fact should be expected, if jumps in volatility are taken

${ }^{1}$ See, in particular, Harrison and Kreps (1979) and Harrison and Pliska (1981, 1983)

${ }^{2}$ Papers documenting this bias include Beckers (1981), Christensen and Prabhala (1998), Fleming (1998), Jorion (1995), Lamoreux and Lastrapes (1993), Amin and Ng (1997) and Canina and Figlewski (1993). 
into account. Also, in general only instantaneous volatility is not affected by a change from the historical to the risk-neutral probability measure, but what is in fact observed is variance over intervals of time. Thus volatility as estimated from time series data does not necessarily coincide with the volatility as required for pricing a derivative. ${ }^{3}$

There is a considerable body of literature dealing with methods to extract the riskneutral distribution from option prices. ${ }^{4}$ Studies applying these methods empirically invariably find that the market puts more weight on extreme events than can be explained by a lognormal distribution, ${ }^{5}$ highlighting the need to depart from the classical Black/Scholes model toward one which can be calibrated to the implied volatility smile, or is at least flexible enough to allow for non-trivial higher order moments, in particular skewness and excess kurtosis.

Following Jarrow and Rudd (1982), Corrado and $\mathrm{Su}(1996)^{6}$ expand the standardised density of the logarithm of the underlying asset price around a standard normal density using a Gram/Charlier Type A series truncated after the fourth order term. This allows one to take into account higher order moments (i.e. skewness and excess kurtosis) of the distribution of the underlying asset when pricing options. Jondeau and Rockinger (2001) show how one can constrain skewness and excess kurtosis in order to ensure that the truncated series is a valid density. However, there is no theoretical reason why one needs to truncate the series after the fourth (rather than some arbitrary other) moment. Rather, since the objective is to obtain an implied distribution which fits observed option prices

\footnotetext{
${ }^{3}$ Similar arguments apply to higher moments of the distribution implied by option prices; see for example the discussion of implied "crash risk" in Bates (2008).

${ }^{4}$ See Bahra (1997) for an overview of the early work in this area. More recently, Haven, Liu, Ma and Shen (2009) took the alternative approach of extracting an implied moment generating function from option prices.

${ }^{5}$ See for example Jackwerth and Rubinstein (1996).

${ }^{6}$ The formula given in Corrado and $\mathrm{Su}$ (1996) contains an error, later corrected by Brown and Robinson (2002) and Jurczenko, Maillet and Negrea (2002).
} 
on a particular day as closely as possible, one might increase the order after which the series is truncated until a perfect fit is achieved. Section 3 below gives an example of this, using a calibration algorithm guaranteeing a valid density at an arbitrary (even) order of truncation. $^{7}$

Probability densities given by Gram/Charlier Type A series expansions are in a way as tractable as the normal density. As in Corrado (2007), European option prices can be expressed in terms of the full (untruncated) series expansion. The present paper will demonstrate that this is also true of a large class of exotic options, involving multiple time points and multiple assets: As Skipper and Buchen (2003) show in the Black/Scholes setting, multiple assets and multiple event dates underlying the payoffs of exotic options can be treated in a general, unified framework. We will see how, by appropriately employing Gram/Charlier Type A series expansions to represent the joint distribution of multiple assets over multiple time horizons, the Skipper/Buchen notation can be applied to derive a generic option pricing formula covering a large class of exotic options in a model consistently calibrated to traded option prices across all available maturities and strikes.

Thus the aim and the contribution of the present paper is methodological rather than empirical. While specific applications of the methods described here are beyond the scope of this paper, in the light of the widespread use of Gram/Charlier Series A option pricing in the empirical literature, ${ }^{8}$ one would expect that the extension to multiple assets and time horizons, along with the algorithm for ensuring valid densities at truncation levels beyond the fourth moment, will prove useful in future empirical analyses.

\footnotetext{
${ }^{7}$ This problem has hitherto been considered intractable in the literature, see e.g. Leon, Mencia and Sentana (2009), who instead propose to use the semi-parametric densities of Gallant and Nychka (1987).

${ }^{8}$ See e.g. Backus, Foresi, Li and Wu (1997), Ane (1999), Jondeau and Rockinger (2000), Navatte and Villa (2000), Capelle-Blancard, Jurczenko and Maillet (2001), Flamouris and Giamouridis (2002), Nikkenin (2003a), Nikkenin (2003b), Jurczenko, Maillet and Negrea (2004), Serna (2004), Valle and Calvo (2005), Vahamaa (2005), Vahamaa, Watzka and Aijo (2005), and Wilkens (2005).
} 
Furthermore, the exotic option formula derived here allows a large class of such options to be priced in a tractable manner in the presence of non-trivial higher order moments of the risk neutral distribution, making the Gram/Charlier Series A approach not only useful for extracting implied risk neutral distributions from liquid option prices, but also for then using those implied distributions to price less liquid exotic options relative to the market.

One should note that this is quite different from — and possibly complementary to another approach of using moment expansions such as Gram/Charlier Series A in quantitative finance, which is to obtain approximate solutions to intractable option pricing problems within existing models. For example, in the standard Black/Scholes model, Airoldi (2005) takes this approach to derive approximate prices for exotic options, for which no closed form solutions are available, by rewriting the option payoff in terms of a new "underlying" stochastic variable, the distribution of which is then represented by a Gram/Charlier series expansion. Similarly, Collin-Dufresne and Goldstein (2002) use an Edgeworth expansion of the probability distribution of the future value of a coupon bond to price swaptions in affine models of the term structure of interest rates. Building on this work, Tanaka, Yamada and Watanabe (2010) use the Gram/Charlier expansion under one forward measure rather than many forward measures and early able to obtain approximate solutions for a larger class of interest-rate- and credit-risk-contingent claims.

The paper is organised as follows. Using the simplest case of a European call option, Section 2 introduces the techniques used for option pricing where the standardised distribution of the logarithmic price of the underlying asset is given by a full Gram/Charlier Type A series expansion. A calibration algorithm ensuring a valid density at an arbitrary (even) order of truncation of the series expansion is described in Section 3. Section 4 provides the results in the fully general setting with multiple assets and multiple event dates. Section 5 discusses implied joint distributions in FX option markets with several currencies and gives an example on market data to demonstrate how the model can be 
used to construct a consistent volatility smile for a cross exchange rate. To facilitate the flow of exposition, proofs have been relegated to Appendix B. Appendix A presents some useful results on Hermite polynomials needed in the paper.

\section{A simple first example: Pricing a European CAll option}

Let us first consider the relatively simple case of a European call option, the price for which under a Gram/Charlier Series A risk neutral density can be found in the existing literature. For example, it is derived by other techniques in Corrado (2007).

As a sufficient condition for the absence of arbitrage for a fixed maturity $T$, we make the following

1. Assumption. The standardised risk-neutral distribution, viewed at a fixed time $t$ for a fixed maturity $T$, of the normalised logarithm $y$ of the asset price $X(T)$ is given by the Gram/Charlier Type A series expansion

$$
\begin{aligned}
f(x) & =\sum_{j=0}^{\infty} c_{j} \operatorname{He}_{j}(x) \phi(x) \\
c_{r} & =\frac{1}{r !} \int_{-\infty}^{\infty} f(x) \operatorname{He}_{r}(x) d x \\
\phi(x) & =\frac{1}{\sqrt{2 \pi}} e^{-\frac{x^{2}}{2}}
\end{aligned}
$$

Interest rates are assumed to be deterministic. ${ }^{9}$

Note that "normalised" refers to translation and scaling such that the resulting random variable has zero mean and unit variance, i.e. the relationship between $X(T)$ and $y$ is given by

$$
X(T)=e^{\sigma y+\mu}
$$

\footnotetext{
${ }^{9}$ Since in this section we are only dealing with a single time horizon, one could alternatively allow for stochastic interest rates by casting Assumption 1 in terms of the distribution under the forward measure. However, the resulting option pricing formula would remain unchanged.
} 
2. Proposition. Under Assumption 1, the risk-neutral expected value $\mu$ of the logarithm of the asset price $X(T)$ must satisfy

$$
\mu=\ln \frac{X(t)}{B(t, T)}-\ln \sum_{j=0}^{\infty} c_{j} \sigma^{j}-\frac{1}{2} \sigma^{2}
$$

where $X(t)$ is the current asset value, $B(t, T)$ is the value at time $t$ of a zero coupon bond maturing in $T$, and $\sigma$ is the standard deviation of $\ln X(T)$.

Note that Proposition 2 corresponds to what Corrado (2007) calls an "explicit martingale restriction" in Gram/Charlier option prices, attributed to Backus, Foresi, Li and Wu (1997), Knight and Satchell (2001) and Kouchard (1999).

3. Proposition. Under Assumption 1, the price of a call option at time $t$ on the asset $X(T)$ with expiry $T$ is given by

$$
\begin{aligned}
C= & B(t, T) \int_{-\infty}^{\infty}\left[e^{\sigma x+\mu}-K\right]^{+} f(x) d x \\
= & X(t) \Phi\left(d^{*}\right)-B(t, T) K \Phi\left(d^{*}-\sigma\right) \\
& +X(t)\left(\sum_{j=0}^{\infty} c_{j} \sigma^{j}\right)^{-1} \phi\left(d^{*}\right) \sum_{j=2}^{\infty} \sum_{i=1}^{j-1} c_{j} \sigma^{i} \mathrm{He}_{j-1-i}\left(-d^{*}+\sigma\right)
\end{aligned}
$$

with

$$
d^{*}=\frac{\mu-\ln K+\sigma^{2}}{\sigma}
$$

\section{A CALibration ALGORIthm ENSURING A VAlid DENSITy}

Given Proposition 3, one can use a set of market option prices for different strikes at a fixed maturity to calibrate the risk-neutral distribution of the underlying asset price. When calculating option prices using (4), the infinite sum is truncated after a finite number of terms. It is well known that in this case, due to the polynomial terms in the Gram/Charlier Series A expansion, conditions need to be imposed on the expansion coefficients $c_{j}$ in $(1)$ 
in order to ensure that $f(x)$ represents a valid probability density with $f(x) \geq 0 \forall x .{ }^{10}$ For truncation after the fourth moment, the case most commonly considered in the literature, ${ }^{11}$ Jondeau and Rockinger (2001) characterise the set of permissible values of $c_{3}$ and $c_{4}$. On the basis of their result, one can constrain the optimisation in the calibration (typically involving the minimisation of some sort of squared distance between market and model prices) to always yield a valid density. Unfortunately, it is not clear how one can extend their approach in a practicable manner to truncated Gram/Charlier expansions involving moments of higher order. Indeed, the number of points required to approximate the domain of valid coefficients would explode. However, this section describes how common well-known unconstrained non-linear optimisation algorithms can be adapted to ensure that the calibrated expansion coefficients in fact yield a valid probability density, without the need to explicitly characterise the domain of permissible coefficients (as a higherdimensional analogue of Jondeau and Rockinger's approach would require). As noted by Leon, Mencia and Sentana (2009), for option pricing applications the non-negativity of the risk neutral density is critical because this ensures the absence of arbitrage within the model.

It always holds that $c_{0}=1$, and we standardise $f(x)$ by setting $c_{1}=0$ and $c_{2}=0$. We truncate the series expansion by setting $c_{j}=0 \forall j>k$ for some choice of $k \geq 4$. Denote by

$$
\mathcal{C}_{k}=\left\{\left(c_{3}, c_{4}, \ldots, c_{k}\right) \in \mathbb{R}^{k-2} \mid f(x) \geq 0 \forall x\right\}
$$

\footnotetext{
${ }^{10}$ This condition is sufficient for $f(x)$ to be a valid density, since

$$
\int_{-\infty}^{\infty} f(x)=1
$$

follows from the properties of Hermite polynomials.

${ }^{11}$ See e.g. Corrado and Su (1996), Bahra (1997), Brown and Robinson (2002) and Jurczenko, Maillet and Negrea (2002).
} 
the set of permissible coefficients when the series is truncated at $k$. Note that if $c_{j} \neq 0$ for $j$ odd, then we must have $c_{j+1} \neq 0$ in order to obtain a valid density. This is because if the highest exponent, say $j$, in the polynomial term of $f(x)$ is odd, for $c_{j}>0$ there exists a $\eta<0$ such that $f(x)<0 \forall x<\eta$, and for $c_{j}<0$ there exists a $\eta>0$ such that $f(x)<0 \forall x>\eta$. Thus, for odd $k, \mathcal{C}_{k}=\mathcal{C}_{k-1}$ (i.e. $c_{k} \equiv 0$ ), and it is therefore sufficient to consider only even $k$.

To carry out the optimisation in $k-2$ dimensions to calibrate the "best fitting" coefficients $\left(c_{3}, c_{4}, \ldots, c_{k}\right)$, one can choose from a large class of optimisation methods involving line minimisations, ${ }^{12}$ which only differ in the manner in which the directions of the line minimisations are determined. This includes Powell's methods as well as conjugate gradient methods. The methods all require a routine (a "line search") to minimise the objective function $g$ along an arbitrary direction $d \in \mathbb{R}^{k-2}$ in the parameter space, i.e. to paraphrase Press, Teukolsky, Vetterling and Flannery (1992), given as input $g$ and the vectors $c^{(0)}, d \in \mathbb{R}^{k-2}$, find the scalar $\lambda$ that minimises $g\left(c^{(0)}+\lambda d\right)$. If we can constrain the line search such that the resulting $c^{(0)}+\lambda d \in \mathcal{C}_{k}$, the optimisation method is ensured to yield a valid density.

To achieve this, note the following properties of the domain of permissible coefficients $\mathcal{C}_{k}:$

4. Lemma. The following statements hold:

a) $\mathcal{C}_{k}$ represents a convex set.

b) Let $x^{(1)}, \ldots, x^{(k)}$ denote the $k$ (possibly complex) roots of the polynomial

$$
\sum_{i=0}^{k} c_{i} \mathrm{He}_{i}(x) .
$$

Then $c \notin \mathcal{C}_{k}$ if and only if for at least one $1 \leq i \leq k, x^{(i)} \in \mathbb{R}^{k-2}$ and $x^{(i)}=x^{(j)}$ for an even number of different $j \neq i$.

\footnotetext{
${ }^{12}$ See Press, Teukolsky, Vetterling and Flannery (1992), Section 10.6 onwards.
} 
It is a consequence of a) that for any $d \in \mathbb{R}^{k-2}$ there exist a $\lambda_{\text {lower }} \in \mathbb{R} \cup\{-\infty\}$ and a $\lambda_{\text {upper }} \in \mathbb{R} \cup\{\infty\}$ such that $c^{(0)}+\lambda d \in \mathcal{C}_{k} \forall \lambda \in\left[\lambda_{\text {lower }}, \lambda_{\text {upper }}\right]$. If one assumes that the domain of permissible coefficients is bounded, ${ }^{13}$ it is a consequence of a) and b) that $\lambda_{\text {lower }}$ and $\lambda_{\text {upper }}$ can easily be identified numerically, i.e. by two runs of a bisection algorithm, which determine, respectively, the smallest $\lambda>0$ and the largest $\lambda<0$ such that for $c^{(1)}=c^{(0)}+\lambda d$, the polynomial

$$
\sum_{i=0}^{k} c_{i}^{(1)} \operatorname{He}_{i}(x)
$$

has at least one real $\operatorname{root}^{14} x^{(i)} \in \mathbb{R}$, and if $x^{(i)}$ is a multiple root of the polynomial, the multiple is odd (this ensures that the polynomial passes through zero, rather than just touching it)..$^{15}$

We can then modify Brent's line search to find a $\lambda_{\min }$ moving us from $c^{(0)}$ to the minimum of the calibration objective function along the direction $d$ while ensuring that $\lambda_{\text {lower }} \leq \lambda_{\text {min }} \leq \lambda_{\text {upper }}$. Once we have this one-dimensional minimisation algorithm along an arbitrary direction $d$, we can implement an optimisation method using line minimisations. For the present paper, Powell's algorithm for minimisation in multiple dimensions was used. ${ }^{16}$

\footnotetext{
${ }^{13}$ For $k=4$, Jondeau and Rockinger (2001) demonstrate that the domain is bounded (not infinite). In the present implementation of the calibration algorithm, $|\lambda|$ is prevented from becoming too large by an arbitrary bound, but in all cases considered so far this arbitrary constraint always turned out to be not binding, suggesting that the domain of permissible constraints is bounded in higher dimensions as well, but an explicit proof of this is not of any practical consequence for the model calibration.

${ }^{14}$ The polynomial roots can easily be calculated using Laguerre's method — see e.g. Press, Teukolsky, Vetterling and Flannery (1992).

${ }^{15}$ Note that an "initial" $c^{(0)}$ in the domain of permissible coefficients can always be found trivially by setting $c_{i}^{(0)}=0 \forall i>0$. The algorithm then ensures that $c^{(0)}$ is in the domain on each subsequent iteration.

${ }^{16}$ For Brent's and Powell's algorithms, again see Press, Teukolsky, Vetterling and Flannery (1992).
} 


\begin{tabular}{|c|c|c|c|c|c|}
\hline Maturity & ATM & 25D RR & 25D BF & 10D RR & 10D BF \\
\hline $1 \mathrm{M}$ & 9.5750 & -0.4500 & 0.2750 & -0.7500 & 1.1250 \\
\hline
\end{tabular}

TABLE 1. USD/EUR at-the-money (ATM) implied volatilities, 25\%-delta (25D) and 10\%-delta (10D) risk reversals (RR) and butterflies (BF) on 24 January 2008. Source: Numerix CrossAsset XL

To illustrate the feasibility of the method for calibration for different levels of truncation of the Gram/Charlier expansion, consider the market data for EUR/USD options with one month time to maturity on 24 January 2008 (Table 1). As is typical in OTC FX options, option values are given as "implied volatility quotes," for at-the-money, and $25 \%$ and $10 \%$ "delta" risk reversals $(\mathrm{RR})$ and butterflies $(\mathrm{BF})$. The "implied volatility" $\sigma_{\mathrm{RR}}$ of a risk reversal is given by the difference of the Black/Scholes implied volatility of an outof-the-money call and put, and the "implied volatility" $\sigma_{\mathrm{BF}}$ of a butterfly is given by the average of the Black/Scholes implied volatilities of the out-of-the-money call and put, minus the at-the-money volatility $\sigma_{\mathrm{ATM}}$.

The "delta" determines the strikes of the out-of-the-money options, which are such that the absolute value of the option delta equals the proscribed level. ${ }^{17}$

Feeding the market data for the maturity of one month into the Powell optimisation, we obtain a reasonable fit for $m=4$, a better fit for $m=6$, and a perfect fit for $m=8$, as Table 2 shows. Figure 1 compares the resulting normalised (i.e. zero mean and unit

\footnotetext{
${ }^{17}$ Characterising strikes by option delta is more informative than absolute strikes or relative "moneyness," as it is primarily determined (though in a non-linear fashion) by how many standard deviations of the distribution of the underlying asset lie between the forward price of the underlying and the strike. Absolute strikes say nothing about whether an option is in, at or out of the money, while relative "moneyness" is the quotient of the current price of the underlying and the strike, which does not take into account volatility.
} 


\begin{tabular}{|c|c|c|c|c|c|}
\hline \multirow[t]{2}{*}{ Strike } & \multirow{2}{*}{$\begin{array}{l}\text { Implied } \\
\text { Volatility }\end{array}$} & \multirow{2}{*}{$\begin{array}{c}\text { Black/Scholes } \\
\text { Price }\end{array}$} & \multicolumn{3}{|c|}{ Fitted Gram/Charlier Price } \\
\hline & & & $m=4$ & $m=6$ & $m=8$ \\
\hline 1.44751 & 0.10075 & 0.0345393 & 0.0345391 & 0.0345243 & 0.0345393 \\
\hline 1.47556 & 0.09575 & 0.0162668 & 0.0162429 & 0.0162719 & 0.0162668 \\
\hline 1.50405 & 0.09625 & 0.0060296 & 0.0060350 & 0.0060288 & 0.0060296 \\
\hline 1.41705 & 0.11075 & 0.0607606 & 0.0608989 & 0.0607624 & 0.0607606 \\
\hline 1.53369 & 0.10325 & 0.0020562 & 0.0020558 & 0.0020563 & 0.0020562 \\
\hline \multicolumn{3}{|c|}{ Sigma } & 0.0296962 & 0.0295336 & 0.0295042 \\
\hline \multicolumn{3}{|c|}{ Skewness } & -0.221359 & -0.143105 & -0.101406 \\
\hline \multicolumn{3}{|c|}{ Excess kurtosis } & 1.65674 & 1.50254 & 1.45571 \\
\hline \multicolumn{3}{|c|}{$c_{5}$} & $\mathrm{~N} / \mathrm{A}$ & 0.00475034 & 0.0106612 \\
\hline \multicolumn{3}{|c|}{$c_{6}$} & $\mathrm{~N} / \mathrm{A}$ & 4.49E-05 & 0.000152405 \\
\hline \multicolumn{3}{|c|}{$c_{7}$} & $\mathrm{~N} / \mathrm{A}$ & $\mathrm{N} / \mathrm{A}$ & 0.000682596 \\
\hline \multicolumn{3}{|c|}{$c_{8}$} & $\mathrm{~N} / \mathrm{A}$ & $\mathrm{N} / \mathrm{A}$ & 0.000114547 \\
\hline
\end{tabular}

TABLE 2. Fit to one-month maturity USD/EUR option data on 24 January 2008.

variance) risk neutral densities with the standard normal density; note the skew and the fatter tails of the implied distribution.

This example is fairly typical of the improved fit achieved by increasing the order after which the Gram/Charlier expansion is truncated. Note that in this example (and in all other example calibrations carried out by the author) the implied distribution remains unimodal even at the higher order of truncation.

\section{Extension to MULtiple time hoRizons AND MUltiple ASSETS}

The modelling of implied risk neutral distributions by Gram/Charlier expansions can be extended to multiple assets and multiple time horizons. Typically a discrete set of time horizons will be given by the maturities of liquidly traded vanilla options to which 


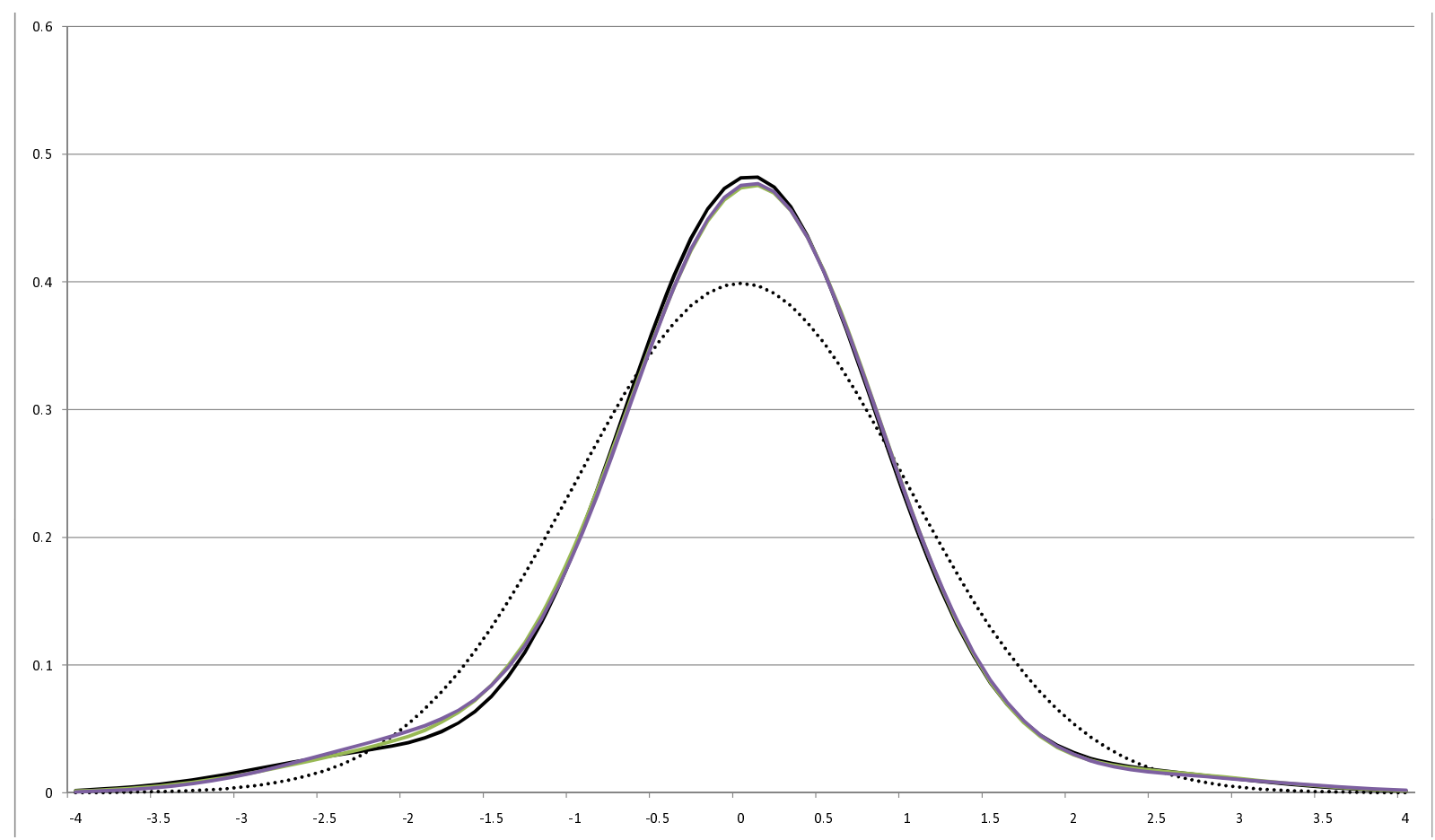

FiguRE 1. Normalised implied risk-neutral distributions (solid lines) fitted to one-month maturity USD/EUR option data on 24 January 2008, vs. the standard normal distribution (dashed line).

the model is calibrated, and the model will need to provide the transition densities for the evolution of asset prices from one time horizon to the next. In particular, it is worth noting that any market information available in reality only determines "averaged volatility to a time horizon" and not "instantaneous volatility." This motivates the following

5. Assumption. The value of the $h$-th asset at time $T_{k}$ can be represented as

$$
X_{h}(0) \exp \left\{\mu_{h, k}+\eta_{h, k} \sum_{j=1}^{d} \sigma_{h, k, j} x_{k, j}\right\}
$$

with

$$
x_{k, j}=\sum_{l=1}^{k} \Delta x_{l, j}
$$

where the increments $\Delta x_{l, j}$ are independently distributed with densities

$$
f_{l, j}(z)=\sum_{m=0}^{\infty} C_{l, j, m} \mathrm{He}_{m}(z) \phi(z)
$$


where for all $l, j, C_{l, j, 0}=1, C_{l, j, 1}=C_{l, j, 2}=0$, i.e. the $\Delta x_{l, j}$ have zero mean and unit variance.

Thus

$C$ is a (time $\times$ factor $\times$ moment $)$ array of coefficients

$\sigma$ is an (asset $\times$ time $\times$ factor) array of factor loadings

$\eta$ is an (asset $\times$ time) array of "volatility levels"

$\mu$ is an (asset $\times$ time) array of "means" under the pricing measure

The model is therefore driven by (and Markovian in) $d$ state variables $x$ (i.e. the value at time $T_{k}$ of the $j$-th state variable is $x_{k, j}$ ), which have transition densities given by Gram/Charlier expansions.

The factor loadings $\sigma$ typically will be chosen in such a way that the factor loading

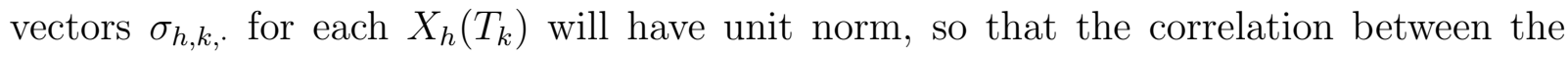
logarithmic increments of two different assets can be expressed as the sum product of the corresponding factor loading vectors, see the construction of a joint distribution in the application example in Section 5. It is therefore convenient to separate the volatility of each asset into volatility levels $\eta$ and (normalised) factor loadings $\sigma$.

6. Proposition. Under Assumption 5, in order to satisfy the no-arbitrage requirement that the discounted asset price processes must be martingales under the risk-neutral measure, the coefficients $\mu_{h, k}$ must satisfy

$$
\mu_{h, k}=\ln \frac{\tilde{B}_{h}\left(0, T_{k}\right)}{B\left(0, T_{k}\right)}-\sum_{j=1}^{d} \sum_{l=1}^{k} \ln \sum_{m=0}^{\infty}\left(\eta_{h, k} \sigma_{h, k, j}\right)^{m} C_{l, j, m}-\frac{1}{2} \eta_{h, k}^{2} \sum_{j=1}^{d} k \sigma_{h, k, j}^{2}
$$

where $B\left(0, T_{k}\right)$ is the value at time 0 of a zero coupon bond maturing in $T_{k}$ and $\tilde{B}_{h}\left(0, T_{k}\right)$ is a "discount factor" embodying the continuous dividend yield of the asset $X_{h} \cdot{ }^{18}$

\footnotetext{
${ }^{18}$ E.g. $\tilde{B}_{h}\left(0, T_{k}\right)=e^{-q T_{k}}$ for a dividend yield of $q$. In the case where $X_{h}$ represents an exchange rate in terms of units of domestic currency per unit of foreign currency, $\tilde{B}_{h}\left(0, T_{k}\right)$ is a foreign zero coupon bond maturing at time $T_{k}$.
} 
In order to treat a large class of exotic payoffs in a unified framework in the Black/Scholes setting, Skipper and Buchen (2003) define what they call an $M$-Binary. Paraphrasing this in our notation, we can state the following

7. Definition. An M-Binary is a derivative financial instrument, the payoff of which depends on the prices of a subset of the assets $X_{h}$ observed at a set of discrete times $T_{k}, k=1, \ldots, K$, and can be represented as

$$
V_{T_{K}}=\underbrace{\left(\prod_{j=1}^{N}\left(X_{\bar{\imath}(j)}\left(T_{\bar{k}(j)}\right)\right)^{\alpha_{j}}\right)}_{\text {payoff amount }} \underbrace{\prod_{l=1}^{M} \mathbb{I}\left\{S_{l} \prod_{j=1}^{N}\left(X_{\bar{\imath}(j)}\left(T_{\bar{k}(j)}\right)\right)^{A_{l, j}}>S_{l} a_{l}\right\}}_{\text {payoff indicator } \bar{I}}
$$

The M-Binary is a European derivative in the sense that payoff is assumed to occur at time $T_{K} . \quad N$ is the payoff dimension, i.e. the number of (asset, time) combinations, on which the payoff of the M-Binary depends. Thus $1 \leq N \leq N \cdot K$ and the index mapping functions $\bar{\imath}(\cdot)$ and $\bar{k}(\cdot)$ serve to select (asset, time) combinations from the $\mathbb{X} \times \mathbb{T}$ set of possible combinations. $M$ is the exercise dimension, i.e. the number of indicator functions of events determining whether a payoff occurs or not. The payoff amount must be representable as a product of powers $\alpha_{j}$ of asset prices. Similarly, the indicator functions condition on the product of (possibly different) powers $A_{j, l}$ of asset prices being greater than some constants $a_{l}$ where this in equality can be reversed by setting $S_{l}=-1$ (instead of $S_{l}=1$ for the "greater than" case).

Assuming deterministic interest rates, ${ }^{19}$ the time zero value of a derivative paying $V_{T_{K}}$ can be calculated as $B\left(0, T_{K}\right) E\left[V_{T_{K}}\right]$, where $B\left(0, T_{K}\right)$ is the time zero price of a zero coupon

\footnotetext{
${ }^{19}$ This assumption can be lifted, but that is left for a follow-on paper.
} 
bond maturing in $T_{K}$. Thus we need to evaluate

$$
\begin{aligned}
E\left[V_{T_{K}}\right] & =E\left[\bar{I} \prod_{j=1}^{N}\left(X_{\bar{\imath}(j)}\left(T_{\bar{k}(j)}\right)\right)^{\alpha_{j}}\right] \\
& =E\left[\bar{I} \prod_{j=1}^{N}\left(X_{\bar{\imath}(j)}(0)\right)^{\alpha_{j}} \exp \left\{\alpha_{j} \mu_{\bar{\imath}(j), \bar{k}(j)}+\alpha_{j} \eta_{\bar{\imath}(j), \bar{k}(j)} \sum_{m=1}^{d} \sigma_{\bar{\imath}(j), \bar{k}(j), m} x_{\bar{k}(j), m}\right\}\right] \\
(11) \quad & =\left(\prod_{j=1}^{N}\left(X_{\bar{\imath}(j)}(0)\right)^{\alpha_{j}} e^{\alpha_{j} \mu_{\bar{\imath}(j), \bar{k}(j)}}\right) E\left[\bar{I} \prod_{j=1}^{N} \exp \left\{\alpha_{j} \eta_{\bar{\imath}(j), \bar{k}(j)} \sum_{m=1}^{d} \sigma_{\bar{\imath}(j), \bar{k}(j), m} x_{\bar{k}(j), m}\right\}\right]
\end{aligned}
$$

Defining

$$
\begin{aligned}
\nu_{l, m} & :=\sum_{j=1}^{N} \alpha_{j} \eta_{\bar{\imath}(j), \bar{k}(j)} \sigma_{\bar{\imath}(j), \bar{k}(j), m} \mathbb{I}\{l \leq \bar{k}(j)\} \\
K & :=\max _{j} \bar{k}(j)
\end{aligned}
$$

Then the latter expectation in (11) can be written as

$$
\begin{aligned}
& E\left[\bar{I} \prod_{j=1}^{N} \exp \left\{\alpha_{j} \eta_{\bar{\imath}(j), \bar{k}(j)} \sum_{m=1}^{d} \sigma_{\bar{\imath}(j), \bar{k}(j), m} x_{\bar{k}(j), m}\right\}\right] \\
& =E\left[\bar{I} \exp \left\{\sum_{m=1}^{d} \sum_{l=1}^{K} \nu_{l, m} \Delta x_{l, m}\right\}\right] \\
& =\int_{-\infty}^{\infty} \cdots \int_{-\infty}^{\infty} \bar{I} \prod_{m=1}^{d} \prod_{l=1}^{K}\left(e^{\nu_{l, m} \Delta x_{l, m}} \sum_{j=0}^{\infty} C_{l, m, j} \operatorname{He}_{j}\left(\Delta x_{l, m}\right) \phi\left(\Delta x_{l, m}\right) d \Delta x_{l, m}\right)
\end{aligned}
$$

and using Lemma 12

$$
=\exp \left\{\frac{1}{2} \sum_{m=1}^{d} \sum_{l=1}^{K} \nu_{l, m}^{2}\right\} \tilde{E}[\bar{I}]
$$

where the densities of the shifted variables $\Delta \tilde{x}_{l, j}=\Delta x_{l, j}-\nu_{l, j}$ under the new measure are given by

$$
\begin{aligned}
& \tilde{f}_{l, j}(z)=\sum_{m=0}^{\infty} \tilde{C}_{l, j, m} \mathrm{He}_{m}(z) \phi(z) \\
& \tilde{C}_{l, j, m}=\sum_{k=m}^{\infty}\left(\begin{array}{c}
k \\
k-m
\end{array}\right) \nu_{l, j}^{k-m} C_{l, j, k}
\end{aligned}
$$


To evaluate $\tilde{E}[\bar{I}]$ where the exercise dimension $M$ is 1 , i.e.

$$
\tilde{E}\left[\mathbb{I}\left\{S_{1} \prod_{j=1}^{N}\left(X_{\bar{\imath}(j)}\left(T_{\bar{k}(j)}\right)\right)^{A_{1, j}}>S_{1} a_{1}\right\}\right]
$$

note that

$$
\begin{aligned}
& \text { (17) } \prod_{j=1}^{N}\left(X_{\bar{\imath}(j)}\left(T_{\bar{k}(j)}\right)\right)^{A_{1, j}} \\
& =\left(\prod_{j=1}^{N}\left(X_{\bar{\imath}(j)}(0)\right)^{A_{1, j}} e^{A_{1, j} \mu_{\bar{\imath}(j), \bar{k}(j)}}\right) \exp \left\{\sum_{j=1}^{N} A_{1, j} \eta_{\bar{\imath}(j), \bar{k}(j)} \sum_{m=1}^{d} \sigma_{\bar{\imath}(j), \bar{k}(j), m} \sum_{l=1}^{\bar{k}(j)}\left(\Delta \tilde{x}_{l, m}+\nu_{l, m}\right)\right\}
\end{aligned}
$$

so what is required is the distribution of a linear combination of $\Delta x_{l, m}$. This is given by the following

8. Lemma. Let the densities of $n$ independent random variables $Z_{k}$ be given by

$$
g_{k}\left(z_{k}\right)=\sum_{j=0}^{\infty} c_{j}^{(k)} \operatorname{He}_{j}\left(z_{k}\right) \phi\left(z_{k}\right)
$$

and define the random variable $\bar{Z}$ by

$$
\bar{Z}:=\sum_{k=1}^{n} \beta_{k} Z_{k}
$$

Then $\bar{Z}$ has the density

$$
\bar{g}(\bar{z})=\sum_{l=0}^{\infty} \bar{c}_{l} \mathrm{He}_{l}\left(\bar{z}\left(\sum_{k=1}^{n} \beta_{k}^{2}\right)^{-\frac{1}{2}}\right) \phi\left(\bar{z}\left(\sum_{k=1}^{n} \beta_{k}^{2}\right)^{-\frac{1}{2}}\right)\left(\sum_{k=1}^{n} \beta_{k}^{2}\right)^{-\frac{1}{2}}
$$

where

$$
\bar{c}_{l}=\left(\sum_{k=1}^{n} \beta_{k}^{2}\right)^{-\frac{1}{2} l} \sum_{j_{1}=0}^{l} \sum_{j_{2}=0}^{l-j_{1}} \ldots \sum_{j_{n-1}=0}^{l-\sum_{h=1}^{n-2} j_{h}}\left(\prod_{k=1}^{n-1} c_{j_{k}}^{(k)} \beta_{k}^{j_{k}}\right) c_{l-\sum_{h=1}^{n-1} j_{h}}^{(n)} \beta_{n}^{l-\sum_{h=1}^{n-1} j_{h}}
$$

Defining

$$
\begin{aligned}
\zeta_{l, m} & :=\sum_{j=1}^{N} A_{1, j} \eta_{\bar{\imath}(j), \bar{k}(j)} \sigma_{\bar{\imath}(j), \bar{k}(j), m} \mathbb{I}\{l \leq \bar{k}(j)\} \\
K & :=\max _{j} \bar{k}(j)
\end{aligned}
$$


(16) can be written as

(21) $\tilde{E}\left[\mathbb{I}\left\{S_{1} \sum_{j=1}^{N} A_{1, j} \eta_{\bar{\imath}(j), \bar{k}(j)} \sum_{m=1}^{d} \sigma_{\bar{\imath}(j), \bar{k}(j), m} \sum_{l=1}^{\bar{k}(j)}\left(\Delta \tilde{x}_{l, m}+\nu_{l, m}\right)\right.\right.$

$$
\left.\left.>S_{1}\left(\ln a_{1}-\sum_{j=1}^{N} A_{1, j}\left(\ln X_{\bar{\imath}(j)}(0)+\mu_{\bar{\imath}(j), \bar{k}(j)}\right)\right)\right\}\right]
$$

$=\tilde{P}\left\{S_{1} \sum_{m=1}^{d} \sum_{l=1}^{K} \zeta_{l, m} \Delta \tilde{x}_{l, m}>S_{1}\left(\ln a_{1}-\sum_{j=1}^{N} A_{1, j}\left(\ln X_{\bar{\imath}(j)}(0)+\mu_{\bar{\imath}(j), \bar{k}(j)}\right)-\sum_{m=1}^{d} \sum_{l=1}^{K} \zeta_{l, m} \nu_{l, m}\right)\right\}$

Further defining

$$
\begin{aligned}
\beta_{k} & =\zeta_{\left[\frac{k-1}{d}\right]+1,(k-1) \% d+1} \\
Z_{k} & =\Delta x_{\left[\frac{k-1}{d}\right]+1,(k-1) \% d+1} \\
\bar{Z} & =\sum_{k=1}^{d \cdot K} \beta_{k} Z_{k} \\
\bar{z}^{*} & =\ln a_{1}-\sum_{j=1}^{N} A_{1, j}\left(\ln X_{\bar{\imath}(j)}(0)+\mu_{\bar{\imath}(j), \bar{k}(j)}\right)-\sum_{m=1}^{d} \sum_{l=1}^{K} \zeta_{l, m} \nu_{l, m}
\end{aligned}
$$

where $\%$ denotes modulo division, this becomes

$$
\tilde{P}\left\{S_{1} \bar{Z}>S_{1} \bar{z}^{*}\right\}
$$

with the coefficients $c_{j}^{(k)}$ of the density $g_{k}($ see $(18))$ given by

$$
c_{j}^{(k)}=\tilde{C}_{\left[\frac{k-1}{d}\right]+1,(k-1) \% d+1, j}
$$

(for $\tilde{C}$, see $(15))$.

We have

$$
\tilde{E}[\bar{I}]=\tilde{P}\left\{S_{1} \bar{Z}>S_{1} \bar{z}^{*}\right\}= \begin{cases}\int_{\bar{z}^{*}}^{\infty} \bar{g}(\bar{z}) d \bar{z} & \text { for } S_{1}=1 \\ \int_{-\infty}^{\bar{z}^{*}} \bar{g}(\bar{z}) d \bar{z} & \text { for } S_{1}=-1\end{cases}
$$

with $\bar{g}(\bar{z})$ given by (19). Setting

$$
z:=\left(\sum_{k=1}^{d \cdot K} \beta_{k}^{2}\right)^{-\frac{1}{2}} \bar{z} \quad z^{*}:=\left(\sum_{k=1}^{d \cdot K} \beta_{k}^{2}\right)^{-\frac{1}{2}} \bar{z}^{*}
$$




\begin{tabular}{|c|c|c|}
\hline$\#$ of assets & \multicolumn{2}{|c|}{2} \\
\hline Payoff dimension $N=$ & \multicolumn{2}{|c|}{2} \\
\hline \# of observation times & \multicolumn{2}{|c|}{1} \\
\hline Exercise dimension $M=$ & \multicolumn{2}{|c|}{1} \\
\hline Asset index mapping $\bar{\imath}(j)=$ & \multicolumn{2}{|c|}{$j$} \\
\hline Time index mapping $\bar{k}(k)=$ & \multicolumn{2}{|c|}{1} \\
\hline & $V^{(1)}$ & $V^{(2)}$ \\
\hline Payoff powers $\alpha$ & $\left(\begin{array}{ll}1 & 0\end{array}\right)$ & $\left(\begin{array}{ll}0 & 1\end{array}\right)$ \\
\hline Sign vector $S$ & $\left(\begin{array}{ll}1 & 1\end{array}\right)$ & $\left(\begin{array}{ll}1 & 1\end{array}\right)$ \\
\hline Indicator powers $A$ & $(1-1)$ & $(1-1)$ \\
\hline Thresholds $a$ & $\kappa_{2} / \kappa_{1}$ & $\kappa_{2} / \kappa_{1}$ \\
\hline
\end{tabular}

TABLE 3. Inputs to calculate the price of options to exchange one asset for another using the $M$-Binary formula.

this becomes

$$
\tilde{E}[\bar{I}]= \begin{cases}\int_{\bar{z}^{*}}^{\infty} \sum_{l=0}^{\infty} \bar{c}_{l} \operatorname{He}_{l}(z) \phi(z) d z & \text { for } S_{1}=1 \\ \int_{-\infty}^{z^{*}} \sum_{l=0}^{\infty} \bar{c}_{l} \operatorname{He}_{l}(z) \phi(z) d z & \text { for } S_{1}=-1\end{cases}
$$

where the $\bar{c}_{l}$ are given by (20). Applying Lemma 13, we have

$$
\tilde{E}[\bar{I}]=\bar{c}_{0} \Phi\left(-S_{1} z^{*}\right)+S_{1} \sum_{l=1}^{\infty} \bar{c}_{l} \phi\left(z^{*}\right) \mathrm{He}_{l-1}\left(z^{*}\right)
$$

Thus we are now in a position to price a number of exotic options, including geometric average options and options to exchange one asset for another (Margrabe (1978) options). The payoff of the latter is given by

$$
\begin{aligned}
& {\left[\kappa_{1} X_{1}\left(T_{1}\right)-\kappa_{2} X_{2}\left(T_{1}\right)\right]^{+}} \\
& \quad=\kappa_{1} X_{1}\left(T_{1}\right) \mathbb{I}\left\{X_{1}\left(T_{1}\right) X_{2}\left(T_{1}\right)^{-1}>\kappa_{2} / \kappa_{1}\right\}-\kappa_{2} X_{2}\left(T_{1}\right) \mathbb{I}\left\{X_{1}\left(T_{1}\right) X_{2}\left(T_{1}\right)^{-1}>\kappa_{2} / \kappa_{1}\right\}
\end{aligned}
$$


i.e. by two $M$-Binaries with parameters as given in Table 3 , which can be inserted into (26) to assemble the time zero value of the above payoff.

\section{Options ON THE CROSS RATE AND THE IMPliEd JOINT DistribUtion.}

Consider a "triangle" of exchange rates, say dollar/yen, dollar/euro and yen/euro. The knowledge of any two of these three exchange rates fixes the third (cross-) rate. For options on these three currency pairs, this does not hold: The volatility of the yen/euro exchange rate is determined by the volatilities of dollar/yen and dollar/euro and the correlation between the two. In the simplest case, if the world were described by the Black/Scholes model, given option prices for all three exchange rates, we could immediately imply the correlation coefficient. In the real world this correlation coefficient will not be constant across strikes - in fact, it is hard to give it any unambiguous meaning for anything but at-the-money options. However, by fitting the joint distribution of the dollar/yen and dollar/euro (and thereby also the yen/euro) exchange rates to all available dollar/yen, dollar/euro and yen/euro FX options, one can extract a meaningful dependence structure (copula) from market prices. Where options on the cross exchange rate are not liquidly traded, the same approach can be used to ensure that the volatility smiles for options on the cross exchange rate are constructed in a consistent, arbitrage-free manner.

Suppose $X_{1}$ and $X_{2}$ are exchange rates with respect to the same base (say, domestic) currency USD, e.g. USD/EUR and USD/JPY. Then the cross exchange rate is given by

$$
X_{3}=\frac{X_{2}}{X_{1}}
$$

eg. EUR/JPY. The price of a call option on JPY with EUR as the base currency can then be written as

$$
B_{\mathrm{EUR}}(t, T) E_{T}^{\mathrm{EUR}}\left[\left[X_{3}(T)-K\right]^{+}\right]
$$


where $E_{T}^{\mathrm{EUR}}$ is the expectation operator under the Euro time $T$ forward measure ${ }^{20}$ and $B_{\mathrm{EUR}}(t, T)$ is the Euro zero coupon bond price. However, if we wish to express cross rate option prices under the domestic (i.e. USD) time $T$ forward measure, we must change measures $^{21}$ and (29) becomes

$$
\begin{aligned}
& B_{\mathrm{EUR}}(t, T) E_{T}^{\mathrm{USD}}\left[\left[X_{3}(T)-K\right]^{+} \frac{B_{\mathrm{EUR}}(T, T) B_{\mathrm{USD}}(t, T) X_{1}(T)}{B_{\mathrm{EUR}}(t, T) B_{\mathrm{USD}}(T, T) X_{1}(t)}\right] \\
= & \frac{B_{\mathrm{USD}}(t, T)}{X_{1}(t)} E_{T}^{\mathrm{USD}}\left[\left[X_{2}(T)-K X_{1}(T)\right]^{+}\right]
\end{aligned}
$$

and thus the option on the cross rate is equivalent to an option (27) to exchange one asset for another.

Suppose that a univariate calibration has been carried out separately for $h=1$ and $h=2$ for

$$
\begin{aligned}
X_{h}\left(T_{k}\right) & =X_{h}(0) \exp \left\{\mu_{h, k}+\eta_{h, k} \bar{z}_{k, h}\right\} \\
\bar{z}_{k, h} & =\sum_{l=1}^{k} \Delta \bar{z}_{l, h}
\end{aligned}
$$

where the $\Delta \bar{z}_{l, h}$ have densities under the domestic risk neutral measure of

$$
g_{l, h}(z)=\sum_{m=0}^{\infty} \bar{C}_{l, h, m} \mathrm{He}_{m}(z) \phi(z)
$$

The univariate calibration will have determined the $\eta_{h, k}$ and $\bar{C}_{l, h, m}$ for $h=1,2$. Now suppose that the distributions of $X_{1}$ and $X_{2}$ under the domestic risk neutral measure are given by Assumption 5. Comparing (32) and (7), we have

$$
\Delta \bar{z}_{l, h}=\sum_{j=1}^{d} \sigma_{h, k, j} \Delta x_{l, j}
$$

\footnotetext{
${ }^{20} \mathrm{As}$ noted previously, in the model at hand stochastic interest rates are not explicitly considered, so the forward and spot risk neutral measures in any given currency can be considered to be identical — the relevant measure change is between risk neutral measures for different currencies.

${ }^{21}$ For the relationship between measures associated with different numeraires, see Geman, El Karoui and Rochet (1995).
} 
Applying Lemma 8, and normalising

$$
\sum_{j=1}^{d} \sigma_{h, k, j}^{2}=1
$$

according to (20) we can express $\bar{C}_{l, h, m}$ in terms of $C_{l, j, m}$ and $\sigma_{h, k, j}$ :

$$
\bar{C}_{l, h, m}=\sum_{j_{1}=0}^{m} \sum_{j_{2}=0}^{m-j_{1}} \cdots \sum_{j_{d-1}=0}^{m-\sum_{n=1}^{d-2}}\left(\prod_{n=1}^{d-1} C_{l, n, j_{n}} \sigma_{h, k, n}^{j_{n}}\right) C_{l, d, m-\sum_{n=1}^{d-1} j_{n}} \sigma_{h, k, d}^{m-\sum_{n=1}^{d-1} j_{n}}
$$

Truncating after the fourth moment (i.e. $\forall l, n, C_{l, n, j=0} \forall j>4$ ), and assuming two factors $(d=2)$, we have for each time step $l$

$$
\begin{aligned}
\bar{C}_{l, h, 0} & =1 \\
\bar{C}_{l, h, 1} & =\sum_{j_{1}=0}^{1} C_{l, 1, j_{1}} \sigma_{h, k, 1}^{j_{1}} C_{l, 2,1-j_{1}} \sigma_{h, k, 2}^{1-j_{1}} \\
& =C_{l, 1,0} C_{l, 2,1} \sigma_{h, k, 2}+C_{l, 1,1} \sigma_{h, k, 1} C_{l, 2,0}=0 \\
\bar{C}_{l, h, 2} & =\sum_{j_{1}=0}^{2} C_{l, 1, j_{1}} \sigma_{h, k, 1}^{j_{1}} C_{l, 2,2-j_{1}} \sigma_{h, k, 2}^{2-j_{1}} \\
& =C_{l, 1,0} C_{l, 2,2} \sigma_{h, k, 2}^{2}+C_{l, 1,1} \sigma_{h, k, 1} C_{l, 2,1} \sigma_{h, k, 2}+C_{l, 1,2} \sigma_{h, k, 1}^{2} C_{l, 2,0}=0 \\
\bar{C}_{l, h, 3} & =\sum_{j_{1}=0}^{3} C_{l, 1, j_{1}} \sigma_{h, k, 1}^{j_{1}} C_{l, 2,3-j_{1}} \sigma_{h, k, 2}^{3-j_{1}} \\
& =C_{l, 2,3} \sigma_{h, k, 2}^{3}+C_{l, 1,3} \sigma_{h, k, 1}^{3} \\
\bar{C}_{l, h, 4} & =\sum_{j_{1}=0}^{4} C_{l, 1, j_{1}} \sigma_{h, k, 1}^{j_{1}} C_{l, 2,4-j_{1}} \sigma_{h, k, 2}^{4-j_{1}} \\
& =C_{l, 2,4} \sigma_{h, k, 2}^{4}+C_{l, 1,4} \sigma_{h, k, 1}^{4}
\end{aligned}
$$

Given $\sigma_{h, k, n}$ and $\bar{C}_{l, 1,3}, \bar{C}_{l, 1,4}, \bar{C}_{l, 2,3}, \bar{C}_{l, 2,4}$, the underlying moments $C_{l, \cdot, \cdot}$ are uniquely determined. Since the $\bar{C}_{.,,,}$are outputs of the univariate calibration for $X_{1}$ and $X_{2}$, it only remains to fix $\sigma_{h, k, n}$. Noting that the correlation between the increments of $\ln X_{1}$ and $\ln X_{2}$ is given by

$$
\rho_{1,2}=\sum_{j=1}^{d} \sigma_{1, k, j} \sigma_{2, k, j}
$$


for $d=2$ we can set

$$
\sigma_{1, k, \cdot}=\left(\begin{array}{l}
1 \\
0
\end{array}\right) \quad \text { and } \quad \sigma_{2, k, \cdot}=\left(\begin{array}{c}
\rho_{1,2} \\
\sqrt{1-\rho_{1,2}^{2}}
\end{array}\right)
$$

We can then proceed to price options on the EUR/JPY cross exchange rate under the USD measure using the formula for an option to exchange one asset for another. Alternatively, we can use the vanilla call option pricing formula after calculating the coefficients $\bar{C}_{l, 3, \text {, of }}$ the distribution of the cross exchange rate under the EUR risk neutral measure:

9. Proposition. Let the USD/EUR and USD/JPY exchange rates $X_{1}$ and $X_{2}$ be given by Assumption 5, where the distribution of the $\Delta x_{l, j}$ is understood to be given under the USD risk neutral measure. Then the cross exchange rate for EUR/JPY is given by

$$
X_{3}=\frac{X_{2}}{X_{1}}
$$

and can be expressed as

$$
X_{3}\left(T_{k}\right)=X_{3}(0) \exp \left\{\hat{\mu}_{3, k}+\hat{\eta}_{3, k} \sum_{l=1}^{k} \Delta \hat{z}_{l, 3}\right\}
$$

with

$$
\begin{aligned}
\hat{\eta}_{3, k} & =\sqrt{\sum_{j=1}^{d}\left(\eta_{2, k} \sigma_{2, k, j}-\eta_{1, k} \sigma_{1, k, j}\right)^{2}} \\
\hat{\mu}_{3, k} & =\ln \frac{B_{J P Y}\left(0, T_{k}\right)}{B_{E U R}\left(0, T_{k}\right)}-\frac{1}{2} \sum_{j=1}^{d} k \hat{\eta}_{3, k}^{2} \hat{\sigma}_{3, k, j}^{2}-\sum_{j=1}^{d} \sum_{l=1}^{k} \ln \sum_{m=0}^{\infty}\left(\hat{\eta}_{3, k} \hat{\sigma}_{3, k, j}\right)^{m} \hat{C}_{l, j, m} \\
\hat{\sigma}_{3, k, j} & =\frac{\eta_{2, k} \sigma_{2, k, j}-\eta_{1, k} \sigma_{1, k, j}}{\hat{\eta}_{3, k}}
\end{aligned}
$$

and where the $\Delta \hat{z}_{l, 3}$ for $l=1, \ldots, k$ are independent random variables under the EUR risk neutral measure with densities

$$
\hat{g}_{l, 3}(z)=\sum_{m=0}^{\infty} \hat{C}_{l, 3, m} \mathrm{He}_{m}(z) \phi(z)
$$




$$
\begin{aligned}
& \hat{C}_{l, 3, m}=\sum_{j_{1}=0}^{m} \sum_{j_{2}=0}^{m-j_{1}} \cdots \sum_{j_{d-1}=0}^{m-\sum_{n=1}^{d-2} j_{n}}\left(\prod_{n=1}^{d-1} \tilde{C}_{l, n, j_{n}} \hat{\sigma}_{3, k, n}^{j_{n}}\right) \tilde{C}_{l, d, m-\sum_{n=1}^{d-1} j_{n}} \hat{\sigma}_{3, k, d}^{m-\sum_{n=1}^{d-1} j_{n}} \\
& \tilde{C}_{l, n, j}=\left(\sum_{m=0}^{\infty}\left(\eta_{1, k} \sigma_{1, k, n}\right)^{m} C_{l, n, m}\right)^{-1} \sum_{m=j}^{\infty}\left(\begin{array}{c}
m \\
m-j
\end{array}\right)\left(\eta_{1, k} \sigma_{1, k, n}\right)^{m-j} C_{l, n, m}
\end{aligned}
$$

In particular, setting $d=2$ and truncating after the fourth moment, we obtain

$$
\hat{C}_{l, 3, m}=\sum_{j_{1}=0}^{m} \tilde{C}_{l, 1, j_{1}} \hat{\sigma}_{3, k, 1}^{j_{1}} \tilde{C}_{l, 2, m-j_{1}} \hat{\sigma}_{3, k, 2}^{m-j_{1}}
$$

i.e.

$$
\begin{gathered}
\hat{C}_{l, 3,0}=1 \quad \hat{C}_{l, 3,1}=\hat{C}_{l, 3,2}=0 \\
\hat{C}_{l, 3, m}=\tilde{C}_{l, 2, m} \hat{\sigma}_{3, k, 2}^{m}+\tilde{C}_{l, 1, m} \hat{\sigma}_{3, k, 1}^{m} \quad m=3,4
\end{gathered}
$$

and

$$
\begin{aligned}
& \tilde{C}_{l, n, 3}=\left(1+\left(\eta_{1, k} \sigma_{1, k, j}\right)^{3} C_{l, n, 3}+\left(\eta_{1, k} \sigma_{1, k, j}\right)^{4} C_{l, n, 4}\right)^{-1}\left(C_{l, n, 3}+4 \eta_{1, k} \sigma_{1, k, j} C_{l, n, 4}\right) \\
& \tilde{C}_{l, n, 4}=\left(1+\left(\eta_{1, k} \sigma_{1, k, j}\right)^{3} C_{l, n, 3}+\left(\eta_{1, k} \sigma_{1, k, j}\right)^{4} C_{l, n, 4}\right)^{-1} C_{l, n, 4}
\end{aligned}
$$

Thus given the correlation between $X_{1}$ and $X_{2}$, the distribution of $X_{3}$ is fully determined. Alternatively, the correlation could be extracted from one option on the cross exchange rate for each maturity. In order to fit the cross smile, a richer structure than the simple additive independent factors in Assumption 5 is required, for example by assuming a truly multivariate Hermite expansion for the joint distribution of the factor increments in each time step.

However, the present approach is nevertheless useful, as it allows two existing FX smiles to be connected in an arbitrage-free, consistent manner to produce the smile for the cross rate. Consider the data for USD/EUR $\left(X_{1}\right)$ and USD/AUD $\left(X_{2}\right)$ options given in Table 4. A perfect fit of the two univariate volatility smiles can be achieved by truncating the Gram/Charlier expansions after order 8, see the calibration results given in Table 5. To determine a consistent risk neutral distribution for the EUR/AUD exchange rate $X_{3}$, a 


\begin{tabular}{|l|c|c|c|c|c|c|}
\hline FX & Maturity & ATM & 25D RR & 25D BF & 10D RR & 10D BF \\
\hline USD/EUR & $1 \mathrm{M}$ & 10.1625 & -0.4325 & 0.2275 & -0.8675 & 0.8225 \\
USD/AUD & $1 \mathrm{M}$ & 11.2750 & -0.8325 & 0.3050 & -1.4825 & 0.9800 \\
\hline
\end{tabular}

TABLE 4. USD/EUR and USD/AUD at-the--money (ATM) implied volatilities, 25\%-delta (25D) and 10\%-delta (10D) risk reversals (RR) and butterflies (BF) on 12 May 2008. Source: Numerix CrossAsset XL

joint distribution of $X_{1}$ and $X_{2}$ consistent with the marginal distributions in Table 5 must be constructed. In this example, in the notation of Assumption 5, we are working with a single time horizon $(k=1)$ and we set the factor loadings

$$
\sigma_{2,1, \cdot}=\left(\begin{array}{c}
1 \\
0
\end{array}\right) \quad \sigma_{1,1, \cdot}=\left(\begin{array}{c}
\rho \\
\sqrt{1-\rho^{2}}
\end{array}\right)
$$

for some correlation coefficient $\rho$. Thus we have two factors, $\Delta x_{1,1}$ and $\Delta x_{1,2}$, and the Gram/Charlier coefficients for the distribution of the first factor are identical to the coefficients of the marginal distribution for $\operatorname{USD} / \operatorname{AUD}\left(X_{2}\right)$. For USD/EUR $\left(X_{1}\right)$, this implies

$$
X_{1}\left(T_{1}\right)=X_{1}(0) \exp \left\{\mu_{1,1}+\eta_{1,1} \sum_{j=1}^{2} \sigma_{1,1, j} \Delta x_{1, j}\right\}
$$

Thus the distribution of $\Delta x_{1,2}$ must be chosen so that the distribution of the left-hand side of (47) matches the marginal distribution for $X_{1}$ already calibrated. By Lemma 8, we have that

$$
\bar{z}=\sum_{j=1}^{2} \sigma_{1,1, j} \Delta x_{1, j}=\rho \Delta x_{1,1}+\sqrt{1-\rho^{2}} \Delta x_{1,2}
$$

has the density

$$
\bar{g}(\bar{z})=\sum_{m=0}^{\infty} \bar{c}_{m} \mathrm{He}_{m}(\bar{z}) \phi(\bar{z})
$$




\begin{tabular}{|c|c|c|c|c|c|c|c|}
\hline \multicolumn{4}{|c|}{ USD/EUR } & \multicolumn{4}{|c|}{ USD/AUD } \\
\hline \multirow[t]{2}{*}{ Strike } & Implied & Black/Scholes & Fitted GC & Strike & Implied & Black/Scholes & Fitted GC \\
\hline & Volatility & Price & Price & & Volatility & Price & Price \\
\hline 1.51845 & 0.10606 & 0.0381345 & 0.0381345 & 0.92379 & 0.11996 & 0.0262261 & 0.0262261 \\
\hline 1.54940 & 0.10163 & 0.0181288 & 0.0181288 & 0.94505 & 0.11275 & 0.0122680 & 0.0122680 \\
\hline 1.58108 & 0.10174 & 0.0066871 & 0.0066871 & 0.96632 & 0.11164 & 0.0044694 & 0.0044694 \\
\hline 1.48612 & 0.11419 & 0.0657190 & 0.0657190 & 0.90132 & 0.12996 & 0.0454237 & 0.0454237 \\
\hline 1.61183 & 0.10551 & 0.0022058 & 0.0022058 & 0.98672 & 0.11514 & 0.0014665 & 0.0014665 \\
\hline \multicolumn{3}{|c|}{ Sigma } & 0.0307062 & \multicolumn{3}{|c|}{ Sigma } & 0.0341475 \\
\hline \multicolumn{3}{|c|}{ Skewness } & -0.231629 & \multicolumn{3}{|c|}{ Skewness } & -0.298006 \\
\hline \multicolumn{3}{|c|}{ Excess kurtosis } & 0.928685 & \multicolumn{3}{|c|}{ Excess kurtosis } & 0.833171 \\
\hline \multicolumn{3}{|c|}{$c_{5}$} & $-5.47 \mathrm{E}-03$ & \multicolumn{3}{|c|}{$c_{5}$} & $-1.34 \mathrm{E}-03$ \\
\hline \multicolumn{3}{|c|}{$c_{6}$} & $-2.35 \mathrm{E}-05$ & \multicolumn{3}{|c|}{$c_{6}$} & $-5.93 \mathrm{E}-05$ \\
\hline \multicolumn{3}{|c|}{$c_{7}$} & $-8.14 \mathrm{E}-04$ & \multicolumn{3}{|c|}{$c_{7}$} & $-8.41 \mathrm{E}-04$ \\
\hline \multicolumn{3}{|c|}{$c_{8}$} & $2.85 \mathrm{E}-04$ & \multicolumn{3}{|c|}{$c_{8}$} & $6.19 \mathrm{E}-04$ \\
\hline
\end{tabular}

TABLE 5. Univariate fits to one--month maturity USD/EUR and USD/AUD option data on 12 May 2008.

with

$$
\bar{c}_{m}=\sum_{j=0}^{m} c_{j}^{(1)} \rho^{j} c_{m-j}^{(2)}\left(\sqrt{1-\rho^{2}}\right)^{m-j}
$$

where the $c_{j}^{(i)}$ are the coefficients of the Gram/Charlier densities of $\Delta x_{1, i}$. The $c_{j}^{(1)}$ are already fixed, and the $\bar{c}_{m}$ are the coefficients of the Gram/Charlier density for the marginal distribution of $X_{1}$. This leaves the $c_{j}^{(2)}$, which therefore must satisfy

$$
c_{m}^{(2)}=\left(\sqrt{1-\rho^{2}}\right)^{-m}\left(\bar{c}_{m}-\sum_{j=1}^{m} c_{j}^{(1)} \rho^{j} c_{m-j}^{(2)}\left(\sqrt{1-\rho^{2}}\right)^{m-j}\right)
$$

and can thus be calculated by iterating forward in $m$ (note that $c_{0}^{(2)}=1, c_{1}^{(2)}=c_{2}^{(2)}=0$ ). 


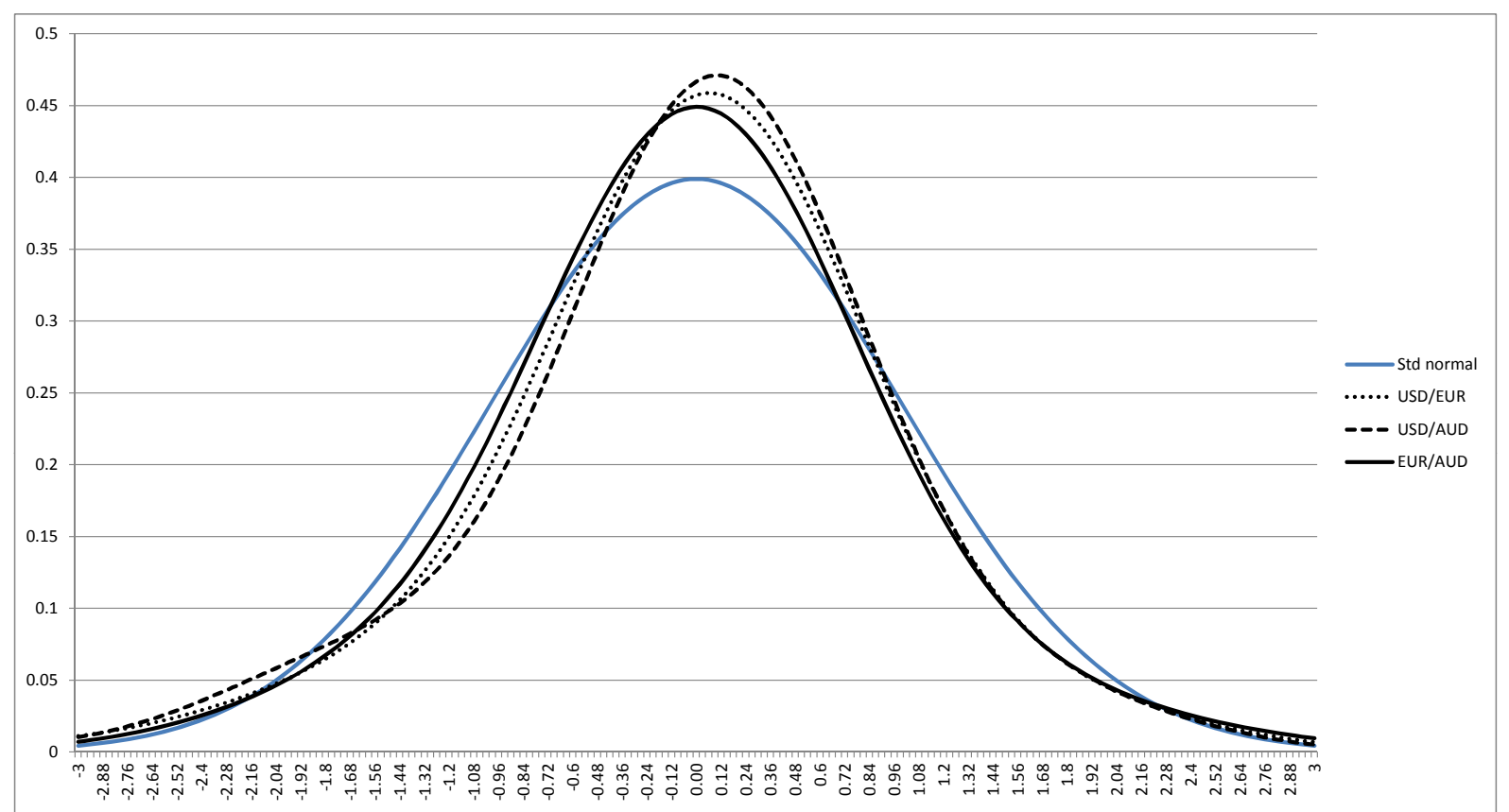

FiguRE 2. Normalised implied risk-neutral distributions fitted to onemonth maturity USD/EUR and USD/AUD option data on 12 May 2008, and the corresponding density for the cross exchange rate EUR/AUD, assuming $\rho=0.5$.

Now we have all the inputs to apply Proposition 9 to yield the coefficients $\hat{C}_{1,3, m}$ of the univariate (marginal) Gram/Charlier density for $X_{3}$, where the "volatility level" is calculated using (41). For an assumed $\rho=0.5$, the normalised (zero mean and unit variance) marginal densities for logarithmic $X_{1}, X_{2}$ and $X_{3}$ (the cross exchange rate) are plotted in Figure 2, along with a standard normal density for comparison. These can then be used to calculate cross-currency option prices (and thus implied volatilities) vi Proposition 3. The different cross-currency volatility smiles resulting from different choices of $\rho$ are plotted in Figure 3. Note that since the cross exchange rate EUR/AUD is given by the quotient of the primary exchange rates USD/AUD and USD/EUR, increasing the correlation between primary exchange rates will decrease the volatility of the cross exchange rate, which is reflected in the graph. 


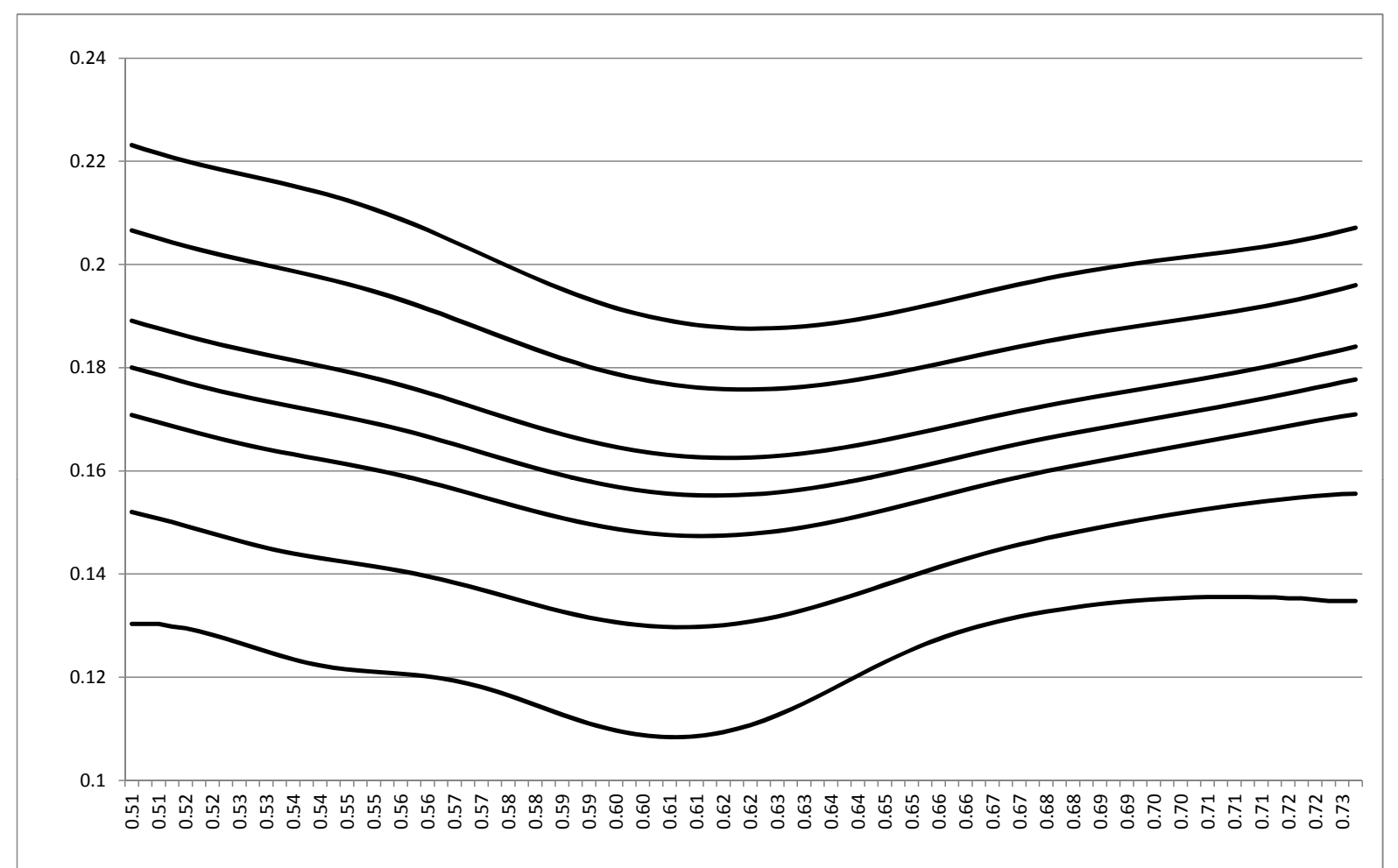

Figure 3. Cross exchange rate (EUR/AUD) implied volatility smiles consistent with one-month maturity USD/EUR and USD/AUD option data on 12 May 2008, for (from top to bottom) $\rho=-0.5,-0.3,-0.1,0,0.1,0.3,0.5$.

\section{Conclusion}

This paper has extended option price modelling based on the risk neutral distributions being given by Gram/Charlier densities to multiple assets and multiple time horizons, while maintaining tractability in the sense that standard - and also a large class of exotic - options can be priced by analytical formulae. These formulae are given at the level of the full (i.e. untruncated) Gram/Charlier expansions, which means that the user can choose an arbitrary level of truncation. Importantly, for any (even) level of truncation, the calibration algorithm presented here guarantees that the truncated expansions represent valid densities. As illustrated by the market data example, choosing a higher order for the truncated expansion allows an ever closer fit of the volatility smile observed in the market. It is worth noting that although the higher order coefficients of the Gram/Charlier expansion also can be interpreted in terms of higher moments of the implied risk neutral 
distribution, this should not necessarily be seen as a statement on the statistical properties of the underlying financial variable. Rather, the implied risk neutral distributions are a convenient way of constructing a (potentially high-dimensional) linear pricing rule to value a large class of exotic options in a manner consistent (in a no-arbitrage sense) with all observed, liquid market prices.

A first illustration of such an application is given by the construction of a EUR/AUD volatility smile that is consistent with the observed USD/EUR and USD/AUD volatility smiles. The extension to multiple assets and multiple time horizons allows a multitude of applications beyond the scope of the present paper, including empirical analysis of joint (both intertemporal and interasset) implied distributions.

\section{Appendix A. Some useful Results on Hermite polynomials under linear}

\section{COORDINATE TRANSFORMS}

In the following, we will use the definition of Hermite polynomials customary in statistics, as given for example in Kendall and Stuart (1969), where they are called "ChebyshevHermite polynomials."

10. Definition. The Hermite polynomials $\mathrm{He}_{i}(\cdot)$ are defined by the identity

$$
(-D)^{i} \phi(x)=\operatorname{He}_{i}(x) \phi(x)
$$

where

$$
D=\frac{d}{d x}
$$

is the differential operator and

$$
\phi(x)=\frac{1}{\sqrt{2 \pi}} e^{-\frac{1}{2} x^{2}}
$$

is the standard normal density function. 
11. Lemma. The Hermite polynomials $\mathrm{He}_{i}(\cdot)$ satisfy

$$
\begin{aligned}
\mathrm{He}_{i}(a x+b) & =\sum_{j=0}^{i}\left(\begin{array}{l}
i \\
j
\end{array}\right) a^{i-j} \mathrm{He}_{i-j}(x) j ! \sum_{m=0}^{\left[\frac{j}{2}\right]} \frac{1}{m ! 2^{m}(j-2 m) !} a^{2 m} \mathrm{He}_{j-2 m}(b) \\
\mathrm{He}_{i}(y+a) & =\sum_{j=0}^{i}\left(\begin{array}{l}
i \\
j
\end{array}\right) \mathrm{He}_{i-j}(y) a^{j} \\
\mathrm{He}_{i}(a x) & =i ! \sum_{m=0}^{\left[\frac{i}{2}\right]} a^{i-2 m} \mathrm{He}_{i-2 m}(x) \frac{\left(a^{2}-1\right)^{m}}{(i-2 m) ! 2^{m} m !}
\end{aligned}
$$

This Lemma is a special case of scaling and translation results well-known in white noise theory, see e.g. Kuo (1996) or Hida, Kuo, Potthoff and Streit (1993). ${ }^{22}$ Similarly, it is highly likely that the other results derived in this section have been been derived and used previously in fields other than mathematical finance. Nevertheless, an accessible presentation is given here for the reader's convenience. ${ }^{23}$

Proof: By virtue of an untruncated Taylor expansion of $\phi$, we have

$$
\begin{aligned}
\phi(t-(a x+b)) & =\sum_{k=0}^{\infty} D^{k} \phi(-(a x+b)) \frac{t^{k}}{k !} \\
& =\sum_{k=0}^{\infty} \operatorname{He}_{k}(-(a x+b))(-1)^{k} \phi(-(a x+b)) \frac{t^{k}}{k !} \\
& =\sum_{k=0}^{\infty} \operatorname{He}_{k}(a x+b) \phi(a x+b) \frac{t^{k}}{k !}
\end{aligned}
$$

and so it holds that

$$
\exp \left\{t(a x+b)-\frac{1}{2} t^{2}\right\}=\sum_{k=0}^{\infty} \operatorname{He}_{k}(a x+b) \frac{t^{k}}{k !}
$$

${ }^{22}$ The author thanks John van der Hoek for pointing this out.

${ }^{23}$ Note also the recurrence relation for Hermite polynomials (see e.g. Abramowitz and Stegun (1964)),

$$
\mathrm{He}_{n+1}(x)=x \mathrm{He}_{n}(x)-n \mathrm{He}_{n-1}(x) .
$$

Other basic properties of Hermite polynomials include

$$
\mathrm{He}_{n}(-x)=(-1)^{n} \mathrm{He}_{n}(x), \quad \mathrm{He}_{2 n+1}(0)=0, \quad n \in \mathbb{N} .
$$


On the other hand, we can write

$$
\begin{aligned}
& \exp \left\{t(a x+b)-\frac{1}{2} t^{2}\right\} \\
= & \exp \left\{t a x-\frac{1}{2} t^{2} a^{2}\right\} \exp \left\{t b-\frac{1}{2} t^{2}\right\} \exp \left\{\frac{1}{2} t^{2} a^{2}\right\} \\
= & \left(\sum_{k=0}^{\infty} \mathrm{He}_{k}(x) \frac{(t a)^{k}}{k !}\right)\left(\sum_{k=0}^{\infty} \mathrm{He}_{k}(b) \frac{t^{k}}{k !}\right)\left(\sum_{k=0}^{\infty} \frac{(t a)^{2 k}}{2^{k} k !}\right) \\
= & \left(\sum_{k=0}^{\infty} \operatorname{He}_{k}(x) \frac{(t a)^{k}}{k !}\right)\left(\sum_{k=0}^{\infty} t^{k} \sum_{m=0}^{\left[\frac{k}{2}\right]} \operatorname{He}_{k-2 m}(b) \frac{1}{(k-2 m) ! 2^{m} m !} a^{2 m}\right) \\
= & \sum_{k=0}^{\infty} t^{k} \sum_{j=0}^{k} \mathrm{He}_{k-j}(x) a^{k-j} \frac{1}{(k-j) !} \sum_{m=0}^{\left[\frac{j}{2}\right]} \operatorname{He}_{j-2 m}(b) \frac{1}{(j-2 m) ! 2^{m} m !} a^{2 m}
\end{aligned}
$$

Comparing the coefficients of $t^{k}$ in (56) and (57) yields (53). Similarly,

$$
\exp \left\{t(y+a)-\frac{1}{2} t^{2}\right\}=\sum_{k=0}^{\infty} \mathrm{He}_{k}(y+a) \frac{t^{k}}{k !}
$$

and

$$
\begin{aligned}
\exp \left\{t(y+a)-\frac{1}{2} t^{2}\right\} & =\exp \left\{t y-\frac{1}{2} t^{2}\right\} \exp \{t a\} \\
& =\left(\sum_{k=0}^{\infty} \operatorname{He}_{k}(y) \frac{t^{k}}{k !}\right)\left(\sum_{k=0}^{\infty} \frac{(t a)^{k}}{k !}\right) \\
& =\sum_{k=0}^{\infty} \frac{t^{k}}{k !} \sum_{m=0}^{k} \operatorname{He}_{k-m}(y) \frac{k !}{m !} \frac{a^{m}}{(k-m) !}
\end{aligned}
$$

so comparing the coefficients of $t^{k}$ in (58) and (59) yields (54). Lastly,

$$
\exp \left\{\operatorname{tax}-\frac{1}{2} t^{2}\right\}=\sum_{k=0}^{\infty} \operatorname{He}_{k}(a x) \frac{t^{k}}{k !}
$$


and

$$
\begin{aligned}
& \exp \left\{t a x-\frac{1}{2} t^{2}\right\} \\
= & \exp \left\{t a x-\frac{1}{2} t^{2} a^{2}\right\} \exp \left\{\frac{1}{2} t^{2} a^{2}\right\} \\
= & \left(\sum_{k=0}^{\infty} \operatorname{He}_{k}(x) \frac{(t a)^{k}}{k !}\right)\left(\sum_{k=0}^{\infty} \frac{\left(t^{2}\left(a^{2}-1\right)\right)^{k}}{2^{k} k !}\right) \\
= & \sum_{k=0}^{\infty} \frac{t^{k}}{k !} \sum_{m=0}^{\left[\frac{k}{2}\right]} k ! \operatorname{He}_{k-2 m}(x) \frac{a^{k-2 m}}{(k-2 m) !} \frac{\left(a^{2}-1\right)^{m}}{2^{m} m !}
\end{aligned}
$$

so comparing the coefficients of $t^{k}$ in (60) and (61) yields (55).

12. Lemma. The following holds:

$$
e^{a z} \sum_{j=0}^{\infty} c_{j} \mathrm{He}_{j}(z) \phi(z)=e^{\frac{1}{2} a^{2}} \sum_{j=0}^{\infty} \tilde{c}_{j} \mathrm{He}_{j}(\tilde{z}) \phi(\tilde{z})
$$

where $\tilde{z}=z-a$ and

$$
\tilde{c}_{j}=\sum_{k=j}^{\infty}\left(\begin{array}{c}
k \\
k-j
\end{array}\right) a^{k-j} c_{k}
$$

Proof:

$$
e^{a z} \sum_{j=0}^{\infty} c_{j} \mathrm{He}_{j}(z) \frac{1}{\sqrt{2 \pi}} e^{-\frac{1}{2} z^{2}}=\frac{1}{\sqrt{2 \pi}} e^{-\frac{1}{2}(z-a)^{2}} e^{\frac{1}{2} a^{2}} \sum_{j=0}^{\infty} c_{j} \mathrm{He}_{j}(z)
$$

and applying (54)

$$
\begin{aligned}
& =\frac{1}{\sqrt{2 \pi}} e^{-\frac{1}{2} \tilde{z}^{2}} e^{\frac{1}{2} a^{2}} \sum_{j=0}^{\infty} c_{j} \sum_{k=0}^{j}\left(\begin{array}{l}
j \\
k
\end{array}\right) \operatorname{He}_{j-k}(\tilde{z}) a^{k} \\
& =e^{\frac{1}{2} a^{2}} \phi(\tilde{z}) \sum_{j=0}^{\infty} \operatorname{He}_{j}(\tilde{z}) \sum_{k=j}^{\infty}\left(\begin{array}{c}
k \\
k-j
\end{array}\right) a^{k-j} c_{k}
\end{aligned}
$$


13. LEMmA. We have for $i \geq 1$

$$
\begin{aligned}
& \int_{a}^{b} \mathrm{He}_{i}(y) \frac{1}{\sqrt{2 \pi}} e^{-\frac{(y-\mu)^{2}}{2}} d y= \\
& \sum_{j=0}^{i-1}\left(\begin{array}{l}
i \\
j
\end{array}\right) \mu^{j}\left(\phi(a-\mu) \mathrm{He}_{i-j-1}(a-\mu)-\phi(b-\mu) \mathrm{He}_{i-j-1}(b-\mu)\right) \\
& +\mu^{i}(\Phi(b-\mu)-\Phi(a-\mu))
\end{aligned}
$$

where $\Phi(\cdot)$ is the cumulative distribution function of the standard normal distribution. If $\mu=0$ and $i \geq 1$

$$
\int_{a}^{b} \mathrm{He}_{i}(y) \frac{1}{\sqrt{2 \pi}} e^{-\frac{y^{2}}{2}} d y=\phi(a) \mathrm{He}_{i-1}(a)-\phi(b) \mathrm{He}_{i-1}(b)
$$

It also follows that if $Y$ is normally distributed with mean $\mu$ and unit variance, we have

$$
E\left[\operatorname{He}_{i}(Y)\right]=\mu^{i}
$$

Proof: Applying Lemma 11 and setting $x:=y-\mu$, we get

$$
\begin{aligned}
& \int_{a}^{b} \operatorname{He}_{i}(y) \frac{1}{\sqrt{2 \pi}} e^{-\frac{(y-\mu)^{2}}{2}} d y \\
= & \int_{a-\mu}^{b-\mu} \frac{1}{\sqrt{2 \pi}} e^{-\frac{x^{2}}{2}}\left(\sum_{j=0}^{i}\left(\begin{array}{l}
i \\
j
\end{array}\right) \operatorname{He}_{i-j}(x) \mu^{j}\right) d x \\
= & \sum_{j=0}^{i}\left(\begin{array}{l}
i \\
j
\end{array}\right) \mu^{j}(-1)^{i-j} \int_{a-\mu}^{b-\mu}\left(D^{i-j} \phi\right)(x) d x \\
= & \sum_{j=0}^{i-1}\left(\begin{array}{l}
i \\
j
\end{array}\right) \mu^{j}(-1)^{i-j}\left(\left(D^{i-j-1} \phi\right)(b-\mu)-\left(D^{i-j-1} \phi\right)(a-\mu)\right)+\mu^{i}(\Phi(b-\mu)-\Phi(a-\mu)) \\
= & \sum_{j=0}^{i-1}\left(\begin{array}{l}
i \\
j
\end{array}\right) \mu^{j}\left(\phi(a-\mu) \operatorname{He}_{i-j-1}(a-\mu)-\phi(b-\mu) \mathrm{He}_{i-j-1}(b-\mu)\right)+\mu^{i}(\Phi(b-\mu)-\Phi(a-\mu))
\end{aligned}
$$

Setting $\mu=0$, this collapses to

$$
\phi(a) \mathrm{He}_{i-1}(a)-\phi(b) \mathrm{He}_{i-1}(b)
$$


By Lemma 11,

(66) $\int_{-\infty}^{\infty} \operatorname{He}_{i}(y) \frac{1}{\sqrt{2 \pi}} e^{-\frac{(y-\mu)^{2}}{2}} d y=\int_{-\infty}^{\infty} \frac{1}{\sqrt{2 \pi}} e^{-\frac{(y-\mu)^{2}}{2}}\left(\sum_{j=0}^{i}\left(\begin{array}{l}i \\ j\end{array}\right) \operatorname{He}_{i-j}(y-\mu) \mu^{j}\right) d(y-\mu)$

Since $E\left[\operatorname{He}_{i}(Y-\mu)\right]=0$ for all $i>0$, and $E\left[\operatorname{He}_{0}(Y-\mu)\right]=1$, (65) follows.

\section{Appendix B. Proofs}

B.1. Proof of Proposition 2. Assumption 1 requires that

$$
\frac{X(t)}{B(t, T)}=\int_{-\infty}^{\infty} e^{\sigma x+\mu} f(x) d x=e^{\mu+\frac{1}{2} \sigma^{2}} \int_{-\infty}^{\infty} \phi(x-\sigma) \sum_{j=0}^{\infty} c_{j} \mathrm{He}_{j}(x) d x
$$

Applying Lemma 13 and solving for $\mu$ yields (3).

\section{B.2. Proof of Proposition 3.}

$$
\int_{-\infty}^{\infty}\left[e^{\sigma x+\mu}-K\right]^{+} f(x) d x=\int_{\frac{\ln K-\mu}{\sigma}}^{\infty} e^{\sigma x+\mu} f(x) d x-K \int_{\frac{\ln K-\mu}{\sigma}}^{\infty} f(x) d x
$$

For the second integral on the RHS, we have

$$
\int_{\frac{\ln K-\mu}{\sigma}}^{\infty} f(x) d x=\int_{\frac{\ln K-\mu}{\sigma}}^{\infty} \sum_{j=0}^{\infty} c_{j} \mathrm{He}_{j}(x) \phi(x) d x
$$

and applying Lemma 13, this becomes

$$
=c_{0} \Phi\left(\frac{\mu-\ln K}{\sigma}\right)+\sum_{j=1}^{\infty} c_{j} \phi\left(\frac{\ln K-\mu}{\sigma}\right) \mathrm{He}_{j-1}\left(\frac{\ln K-\mu}{\sigma}\right)
$$

For the first integral on the RHS of (68), we have

$$
\int_{\frac{\ln K-\mu}{\sigma}}^{\infty} e^{\sigma x+\mu} f(x) d x=e^{\mu+\frac{1}{2} \sigma^{2}} \int_{\frac{\ln K-\mu}{\sigma}}^{\infty} \phi(x-\sigma) \sum_{j=0}^{\infty} c_{j} \mathrm{He}_{j}(x) d x
$$


and by Lemma 13 this is

$$
\begin{aligned}
=e^{\mu+\frac{1}{2} \sigma^{2}} & \sum_{j=0}^{\infty} c_{j}\left(\sigma^{j} \Phi\left(\frac{\mu-\ln K+\sigma^{2}}{\sigma}\right)\right. \\
& \left.+\sum_{i=0}^{j-1}\left(\begin{array}{l}
j \\
i
\end{array}\right) \sigma^{i} \phi\left(\frac{\ln K-\mu-\sigma^{2}}{\sigma}\right) \operatorname{He}_{j-i-1}\left(\frac{\ln K-\mu-\sigma^{2}}{\sigma}\right)\right)
\end{aligned}
$$

Noting from Proposition 2 that

$$
\frac{X(t)}{B(t, T)}=e^{\mu+\frac{1}{2} \sigma^{2}} \sum_{j=0}^{\infty} c_{j} \sigma^{j}
$$

(72) becomes

$$
\begin{aligned}
& \int_{\frac{\ln K-\mu}{\sigma}}^{\infty} e^{\sigma x+\mu} f(x) d x \\
= & \frac{X(t)}{B(t, T)}\left(\Phi\left(\frac{\mu-\ln K+\sigma^{2}}{\sigma}\right)\right. \\
& \left.+\left(\sum_{j=0}^{\infty} c_{j} \sigma^{j}\right)^{-1} \sum_{j=1}^{\infty} c_{j} \sum_{i=0}^{j-1}\left(\begin{array}{l}
j \\
i
\end{array}\right) \sigma^{i} \phi\left(\frac{\ln K-\mu-\sigma^{2}}{\sigma}\right) \operatorname{He}_{j-i-1}\left(\frac{\ln K-\mu-\sigma^{2}}{\sigma}\right)\right)
\end{aligned}
$$

Furthermore,

$$
\begin{aligned}
& K \sum_{j=1}^{\infty} c_{j} \phi\left(\frac{\ln K-\mu}{\sigma}\right) \mathrm{He}_{j-1}\left(\frac{\ln K-\mu}{\sigma}\right) \\
= & K e^{\mu+\frac{1}{2} \sigma^{2}} \sum_{j=1}^{\infty} c_{j} \mathrm{He}_{j-1}\left(\frac{\ln K-\mu}{\sigma}\right) \frac{1}{\sqrt{2 \pi} \sigma} \exp \left\{-\frac{1}{2 \sigma^{2}}\left((\ln K-\mu)^{2}+2 \sigma^{2} \mu+\sigma^{4}\right)\right\} \\
= & \left.K e^{\mu+\frac{1}{2} \sigma^{2}} e^{-\ln K} \sum_{j=1}^{\infty} c_{j} \mathrm{He}_{j-1}\left(\frac{\ln K-\mu}{\sigma}\right) \frac{1}{\sqrt{2 \pi} \sigma} \exp \left\{-\frac{1}{2 \sigma^{2}}\left(\mu-\ln K+\sigma^{2}\right)^{2}\right)\right\}
\end{aligned}
$$

and again applying Lemma 11

$$
=e^{\mu+\frac{1}{2} \sigma^{2}} \sum_{j=1}^{\infty} c_{j} \sum_{i=0}^{j-1}\left(\begin{array}{c}
j-1 \\
i
\end{array}\right) \operatorname{He}_{j-1-i}\left(\frac{\ln K-\mu-\sigma^{2}}{\sigma}\right) \sigma^{i} \phi\left(\frac{\mu-\ln K+\sigma^{2}}{\sigma}\right)
$$

Substituting (70), (74) and (75) into (68) and noting that

$$
c_{0}=1 \quad\left(\begin{array}{l}
1 \\
0
\end{array}\right)-\left(\begin{array}{l}
0 \\
0
\end{array}\right)=0 \quad \phi(x)=\phi(-x)
$$


and

$$
\begin{array}{ll}
\left(\begin{array}{l}
j \\
0
\end{array}\right)-\left(\begin{array}{c}
j-1 \\
0
\end{array}\right)=0 & \forall j \geq 1 \\
\left(\begin{array}{l}
j \\
i
\end{array}\right)-\left(\begin{array}{c}
j-1 \\
i
\end{array}\right)=\left(\begin{array}{c}
j-1 \\
i-1
\end{array}\right) & \forall j, i \geq 1
\end{array}
$$

we get

$$
\begin{aligned}
C= & X(t) \Phi\left(d^{*}\right)-B(t, T) K \Phi\left(d^{*}-\sigma\right) \\
& +X(t)\left(\sum_{j=0}^{\infty} c_{j} \sigma^{j}\right)^{-1} \sum_{j=2}^{\infty} \sum_{i=1}^{j-1}\left(\begin{array}{l}
j-1 \\
i-1
\end{array}\right) c_{j} \sigma^{i} \phi\left(d^{*}\right) \mathrm{He}_{j-1-i}\left(-d^{*}\right)
\end{aligned}
$$

Applying Lemma 11 to replace $\mathrm{He}_{j-1-i}\left(-d^{*}\right)$ by $\mathrm{He}_{j-1-i}\left(-d^{*}+\sigma\right)$ and noting that

$$
\sum_{k=0}^{n-1}\left(\begin{array}{c}
j-1-(n-k) \\
k
\end{array}\right)=\left(\begin{array}{l}
j-1 \\
n-1
\end{array}\right)
$$

yields (4).

B.3. Proof of Lemma 4. If the coefficient vectors $c^{(1)}$ and $c^{(2)}$ are elements of $\mathcal{C}_{k}$, i.e.

$$
\sum_{i=0}^{k} c_{i}^{(1)} \mathrm{He}_{i}(x) \geq 0 \quad \text { and } \quad \sum_{i=0}^{k} c_{i}^{(2)} \mathrm{He}_{i}(x) \geq 0 \quad \forall x
$$

then it immediately follows that for any $0 \leq \alpha \leq 1$

$$
\sum_{i=0}^{k}\left(\alpha c_{i}^{(1)}+(1-\alpha) c_{i}^{(2)}\right) \operatorname{He}_{i}(x) \geq 0 \quad \forall x
$$

i.e. $\alpha c^{(1)}+(1-\alpha) c^{(2)} \in \mathcal{C}_{k}$, i.e $\mathcal{C}_{k}$ is a convex set.

Furthermore, by the properties of Hermite polynomials, the polynomial

$$
\sum_{i=0}^{k} c_{i} \mathrm{He}_{i}(x)
$$

will be positive for at least some $x$. Therefore, $c \notin \mathcal{C}_{k}$ if and only if the polynomial (77) passes through zero (as opposed to just touching it). This will be the case if and only if (77) has at least one real root $x^{(i)} \in \mathbb{R}$, and if $x^{(i)}$ is a multiple root of the polynomial, the multiple is odd. 
B.4. Proof of Proposition 6. In order for Assumption 5 to be consistent with the absence of arbitrage, we must have

$$
\begin{aligned}
\frac{X_{h}(0) \tilde{B}_{h}\left(0, T_{k}\right)}{B\left(0, T_{k}\right)}=E\left[X_{h}(0) \exp \left\{\mu_{h, k}+\eta_{h, k} \sum_{j=1}^{d} \sigma_{h, k, j} x_{k, j}\right\}\right] \\
=X_{h}(0) \int_{-\infty}^{\infty} \cdots \int_{-\infty}^{\infty} \exp \left\{\mu_{h, k}+\eta_{h, k} \sum_{j=1}^{d} \sigma_{h, k, j} \sum_{l=1}^{k} \Delta x_{l, j}\right\} \\
\prod_{j=1}^{d} \prod_{l=1}^{k}\left(f_{l, j}\left(\Delta x_{l, j}\right) d \Delta x_{l, j}\right)
\end{aligned}
$$

This is equivalent to

$$
e^{-\mu_{h, k}}=\frac{B\left(0, T_{k}\right)}{\tilde{B}_{h}\left(0, T_{k}\right)} \int_{-\infty}^{\infty} \cdots \int_{-\infty}^{\infty} \prod_{j=1}^{d} \prod_{l=1}^{k}\left(\exp \left\{\eta_{h, k} \sigma_{h, k, j} \Delta x_{l, j}\right\} f_{l, j}\left(\Delta x_{l, j}\right) d \Delta x_{l, j}\right)
$$

Consider

$$
\begin{aligned}
& \int_{-\infty}^{\infty} \exp \left\{\eta_{h, k} \sigma_{h, k, j} \Delta x_{l, j}\right\} f_{l, j}\left(\Delta x_{l, j}\right) d \Delta x_{l, j} \\
= & \exp \left\{\frac{1}{2} \eta_{h, k}^{2} \sigma_{h, k, j}^{2}\right\} \int_{-\infty}^{\infty} \phi\left(\Delta x_{l, j}-\eta_{h, k} \sigma_{h, k, j}\right) \sum_{m=0}^{\infty} C_{l, j, m} \mathrm{He}_{m}\left(\Delta x_{l, j}\right) d \Delta x_{l, j}
\end{aligned}
$$

and applying Lemma 13

$$
=\exp \left\{\frac{1}{2} \eta_{h, k}^{2} \sigma_{h, k, j}^{2}\right\} \sum_{m=0}^{\infty} C_{l, j, m}\left(\eta_{h, k} \sigma_{h, k, j}\right)^{m}
$$

Inserting this into (78) yields (9).

B.5. Proof of Lemma 8. The characteristic function corresponding to the density $g_{k}$ is

$$
\begin{aligned}
\psi_{k}(t) & =\int_{-\infty}^{\infty} e^{i t z_{k}} g_{k}\left(z_{k}\right) d z_{k} \\
& =\sum_{j=0}^{\infty} c_{j}^{(k)} \int_{-\infty}^{\infty} e^{i t z_{k}} \operatorname{He}_{j}\left(z_{k}\right) \phi\left(z_{k}\right) d z_{k}
\end{aligned}
$$


Noting that $(-D)^{j} \phi\left(z_{k}\right)=\mathrm{He}_{j}\left(z_{k}\right) \phi\left(z_{k}\right)$, we can apply integration by parts, i.e.

$$
\begin{aligned}
\int_{-\infty}^{\infty} e^{i t z_{k}} \mathrm{He}_{j}\left(z_{k}\right) \phi\left(z_{k}\right) d z_{k} & =\int_{-\infty}^{\infty} e^{i t z_{k}}(-D)^{j} \phi\left(z_{k}\right) d z_{k} \\
& =-\left.e^{i t z_{k}}(-D)^{j-1} \phi\left(z_{k}\right)\right|_{-\infty} ^{\infty}+\int_{-\infty}^{\infty} i t e^{i t z_{k}}(-D)^{j-1} \phi\left(z_{k}\right) d z_{k} \\
& =\underbrace{-\left.e^{i t z_{k}} \mathrm{He}_{j-1}\left(z_{k}\right) \phi\left(z_{k}\right)\right|_{-\infty} ^{\infty}}_{=0}+\int_{-\infty}^{\infty} i t e^{i t z_{k}}(-D)^{j-1} \phi\left(z_{k}\right) d z_{k}
\end{aligned}
$$

Applying this $j$ times, we are left with

$$
=(i t)^{j} \int_{-\infty}^{\infty} e^{i t z_{k}} \phi\left(z_{k}\right) d z_{k}
$$

where the remaining integral is the well known characteristic function of the standard normal distribution, i.e.

$$
=(i t)^{j} e^{-\frac{1}{2} t^{2}}
$$

and so we have

$$
\psi_{k}(t)=\sum_{j=0}^{\infty} c_{j}^{(k)} e^{-\frac{1}{2} t^{2}}(i t)^{j}
$$

and thus the characteristic function of the random variable $\bar{Z}$ is

$$
\begin{aligned}
& \bar{\psi}(t)=\prod_{k=1}^{n}\left(\sum_{j=0}^{\infty} c_{j}^{(k)} e^{-\frac{1}{2} \beta_{k}^{2} t^{2}}\left(i \beta_{k} t\right)^{j}\right) \\
& =\sum_{j_{1}=0}^{\infty} \cdots \sum_{j_{n}=0}^{\infty} \prod_{k=1}^{n} c_{j_{k}}^{(k)} e^{-\frac{1}{2} \beta_{k}^{2} t^{2}}\left(i \beta_{k} t\right)^{j_{k}} \\
& =\exp \left\{-\frac{1}{2} \sum_{k=1}^{n} \beta_{k}^{2} t^{2}\right\} \sum_{j_{1}=0}^{\infty} \cdots \sum_{j_{n}=0}^{\infty}(i t)^{\sum_{k=1}^{n} j_{k}} \prod_{k=1}^{n} c_{j_{k}}^{(k)} \beta_{k}^{j_{k}} \\
& =\exp \left\{-\frac{1}{2} \sum_{k=1}^{n} \beta_{k}^{2} t^{2}\right\} \sum_{l=0}^{\infty}\left(i t\left(\sum_{k=1}^{n} \beta_{k}^{2}\right)^{\frac{1}{2}}\right)^{l}\left(\sum_{k=1}^{n} \beta_{k}^{2}\right)^{-\frac{1}{2} l} \\
& \sum_{j_{1}=0}^{l} \sum_{j_{2}=0}^{l-j_{1}} \ldots \sum_{j_{n-1}=0}^{l-\sum_{h=1}^{n-2} j_{h}}\left(\prod_{k=1}^{n-1} c_{j_{k}}^{(k)} \beta_{k}^{j_{k}}\right) c_{l-\sum_{h=1}^{n-1} j_{h}}^{(n)} \beta_{n}^{l-\sum_{h=1}^{n-1} j_{h}}
\end{aligned}
$$

and by comparing coefficients with (80) it is evident that (19) holds. 
B.6. Proof of Proposition 9. Under Assumption 5, the joint density of the factor increments $\Delta x_{l, j}$ under the USD risk neutral measure is given as

$$
\prod_{j=1}^{d} \prod_{l=1}^{k} f_{l, j}\left(\Delta x_{l, j}\right)
$$

The Radon/Nikodym derivative relating the USD and EUR risk neutral measures for time horizon $T_{k}$ is

$$
\frac{d P_{T_{k}}^{\mathrm{EUR}}}{d P_{T_{k}}^{\mathrm{USD}}}=\frac{B_{\mathrm{EUR}}\left(T_{k}, T_{k}\right) B_{\mathrm{USD}}\left(0, T_{k}\right) X_{1}\left(T_{k}\right)}{B_{\mathrm{EUR}}\left(0, T_{k}\right) B_{\mathrm{USD}}\left(T_{k}, T_{k}\right) X_{1}(0)}
$$

Thus the density of the $\Delta x_{l, j}$ under the EUR risk neutral measure is

$$
\begin{aligned}
&\left(\prod_{j=1}^{d} \prod_{l=1}^{k} f_{l, j}\left(\Delta x_{l, j}\right)\right) \frac{d P_{T_{k}}^{\mathrm{EUR}}}{d P_{T_{k}}^{\mathrm{USD}}} \\
&= \frac{B_{\mathrm{USD}}\left(0, T_{k}\right)}{B_{\mathrm{EUR}}\left(0, T_{k}\right) X_{1}(0)} X_{1}\left(T_{k}\right) \prod_{j=1}^{d} \prod_{l=1}^{k} f_{l, j}\left(\Delta x_{l, j}\right) \\
&= \frac{B_{\mathrm{USD}}\left(0, T_{k}\right)}{B_{\mathrm{EUR}}\left(0, T_{k}\right)} e^{\mu_{1, k}}\left(\prod_{j=1}^{d} \prod_{l=1}^{k} \exp \left\{\eta_{1, k} \sigma_{1, k, j} \Delta x_{l, j}\right\}\right) \prod_{j=1}^{d} \prod_{l=1}^{k} f_{l, j}\left(\Delta x_{l, j}\right) \\
&= \exp \left\{-\sum_{j=1}^{d} \sum_{l=1}^{k} \ln \sum_{m=0}^{\infty}\left(\eta_{1, k} \sigma_{1, k, j}\right)^{m} C_{l, j, m}-\frac{1}{2} \eta_{1, k}^{2} \sum_{j=1}^{d} k \sigma_{1, k, j}^{2}\right\} \\
& \prod_{j=1}^{d} \prod_{l=1}^{k} \exp \left\{\eta_{1, k} \sigma_{1, k, j} \Delta x_{l, j}\right\} f_{l, j}\left(\Delta x_{l, j}\right)
\end{aligned}
$$

By Lemma 12 this equals

$$
\begin{aligned}
\exp \left\{-\sum_{j=1}^{d} \sum_{l=1}^{k} \ln \sum_{m=0}^{\infty}\left(\eta_{1, k} \sigma_{1, k, j}\right)^{m} C_{l, j, m}-\frac{1}{2} \eta_{1, k}^{2} \sum_{j=1}^{d} k \sigma_{1, k, j}^{2}\right\} & \\
& \prod_{j=1}^{d} \prod_{l=1}^{k} \exp \left\{\frac{1}{2} \eta_{1, k}^{2} \sigma_{1, k, j}^{2}\right\} \sum_{m=0}^{\infty} C_{l, j, m}^{\prime} \mathrm{He}_{m}\left(\Delta \tilde{x}_{l, j}\right) \phi\left(\Delta \tilde{x}_{l, j}\right)
\end{aligned}
$$


with

$$
\begin{aligned}
\Delta \tilde{x}_{l, j} & =\Delta x_{l, j}-\eta_{1, k} \sigma_{1, k, j} \\
C_{l, j, m}^{\prime} & =\sum_{n=m}^{\infty}\left(\begin{array}{c}
n \\
n-m
\end{array}\right)\left(\eta_{1, k} \sigma_{1, k, j}\right)^{n-m} C_{l, j, n}
\end{aligned}
$$

Note that

$$
\int_{-\infty}^{\infty} \sum_{m=0}^{\infty} C_{l, j, m}^{\prime} \operatorname{He}_{m}\left(\Delta \tilde{x}_{l, j}\right) \phi\left(\Delta \tilde{x}_{l, j}\right) d\left(\Delta \tilde{x}_{l, j}\right)=C_{l, j, 0}^{\prime}=\sum_{n=0}^{\infty}\left(\begin{array}{c}
n \\
n-0
\end{array}\right)\left(\eta_{1, k} \sigma_{1, k, j}\right)^{n} C_{l, j, n}
$$

Thus we normalise

$$
\tilde{C}_{l, j, m}=\frac{C_{l, j, m}^{\prime}}{C_{l, j, 0}^{\prime}}
$$

and we have

$$
\left(\prod_{j=1}^{d} \prod_{l=1}^{k} f_{l, j}\left(\Delta x_{l, j}\right)\right) \frac{d P_{T_{k}}^{\mathrm{EUR}}}{d P_{T_{k}}^{\mathrm{USD}}}=\prod_{j=1}^{d} \prod_{l=1}^{k} \hat{f}_{l, j}\left(\Delta \tilde{x}_{l, j}\right)
$$

with

$$
\hat{f}_{l, j}\left(\Delta \tilde{x}_{l, j}\right)=\sum_{m=0}^{\infty} \tilde{C}_{l, j, m}^{\prime} \operatorname{He}_{m}\left(\Delta \tilde{x}_{l, j}\right) \phi\left(\Delta \tilde{x}_{l, j}\right)
$$

$\tilde{C}_{l, j, m}$ and $\Delta \tilde{x}_{l, j}$ defined as above. Under Assumption 5 , it then follows from $X_{3}=X_{2} / X_{1}$ that

$$
\begin{aligned}
X_{3}\left(T_{k}\right) & =X_{3}(0) \exp \left\{\mu_{2, k}-\mu_{1, k}+\sum_{j=1}^{k}\left(\eta_{2, k} \sigma_{2, k, j}-\eta_{1, k} \sigma_{1, k, j}\right) \sum_{l=1}^{k}\left(\Delta \tilde{x}_{l, j}+\eta_{1, k} \sigma_{1, k, j}\right)\right\} \\
& =X_{3}(0) \exp \left\{\hat{\mu}_{3, k}+\hat{\eta}_{3, k} \sum_{l=1}^{k} \Delta \hat{z}_{l, 3}\right\}
\end{aligned}
$$

with

$$
\begin{aligned}
\Delta \hat{z}_{l, 3} & =\sum_{j=1}^{d} \hat{\sigma}_{3, k, j} \Delta \tilde{x}_{l, j} \\
\hat{\sigma}_{3, k, j} & =\frac{\eta_{2, k} \sigma_{2, k, j}-\eta_{1, k} \sigma_{1, k, j}}{\hat{\eta}_{3, k}} \\
\hat{\eta}_{3, k} & =\sqrt{\sum_{j=1}^{d}\left(\eta_{2, k} \sigma_{2, k, j}-\eta_{1, k} \sigma_{1, k, j}\right)^{2}}
\end{aligned}
$$


(44) then follows from Lemma 8. Lastly,

$$
\begin{aligned}
\hat{\mu}_{3, k}= & \mu_{2, k}-\mu_{1, k}+\sum_{j=1}^{d}\left(\eta_{2, k} \sigma_{2, k, j}-\eta_{1, k} \sigma_{1, k, j}\right) k \eta_{1, k} \sigma_{1, k, j} \\
= & \ln \frac{\tilde{B}_{2}\left(0, T_{k}\right)}{\tilde{B}_{1}\left(0, T_{k}\right)}-\sum_{j=1}^{d} \sum_{l=1}^{k} \ln \sum_{m=0}^{\infty} C_{l, j, m}\left(\eta_{2, k} \sigma_{2, k, j}\right)^{m}+\sum_{j=1}^{d} \sum_{l=1}^{k} \ln \sum_{m=0}^{\infty} C_{l, j, m}\left(\eta_{1, k} \sigma_{1, k, j}\right)^{m} \\
& -\frac{1}{2} \eta_{2, k}^{2} \sum_{j=1}^{d} k \sigma_{2, k, j}^{2}+\frac{1}{2} \eta_{1, k}^{2} \sum_{j=1}^{d} k \sigma_{1, k, j}^{2}+\sum_{j=1}^{d}\left(\eta_{2, k} \sigma_{2, k, j}-\eta_{1, k} \sigma_{1, k, j}\right) k \eta_{1, k} \sigma_{1, k, j} \\
= & \ln \frac{B_{\mathrm{JPY}}\left(0, T_{k}\right)}{B_{\mathrm{EUR}}\left(0, T_{k}\right)}-\frac{1}{2} \sum_{j=1}^{d} k(\underbrace{\eta_{2, k} \sigma_{2, k, j}-\eta_{1, k} \sigma_{1, k, j}}_{=\hat{\eta}_{3, k}^{2} \hat{\sigma}_{3, k, j}^{2}})^{2} \\
& -\sum_{j=1}^{d} \sum_{l=1}^{k} \ln \sum_{m=0}^{\infty} C_{l, j, m}\left(\eta_{2, k} \sigma_{2, k, j}\right)^{m}+\sum_{j=1}^{d} \sum_{l=1}^{k} \ln \sum_{m=0}^{\infty} C_{l, j, m}\left(\eta_{1, k} \sigma_{1, k, j}\right)^{m}
\end{aligned}
$$

To obtain (42), the following must hold:

$$
\ln \sum_{m=0}^{\infty} C_{l, j, m}\left(\eta_{2, k} \sigma_{2, k, j}\right)^{m}-\ln \sum_{m=0}^{\infty} C_{l, j, m}\left(\eta_{1, k} \sigma_{1, k, j}\right)^{m}=\ln \sum_{m=0}^{\infty} \hat{C}_{l, j, m}\left(\hat{\eta}_{3, k} \hat{\sigma}_{3, k, j}\right)^{m}
$$

Substituting $\hat{C}_{l, j, m}$ as it is defined in the proposition, note that

$$
\begin{aligned}
& \sum_{m=0}^{\infty} \hat{C}_{l, j, m}\left(\hat{\eta}_{3, k} \hat{\sigma}_{3, k, j}\right)^{m} \\
= & \left(\sum_{m=0}^{\infty} C_{l, j, m}\left(\eta_{1, k} \sigma_{1, k, j}\right)^{m}\right)^{-1} \sum_{m=0}^{\infty}\left(\hat{\eta}_{3, k} \hat{\sigma}_{3, k, j}\right)^{m} \sum_{n=m}^{\infty}\left(\begin{array}{c}
n \\
n-m
\end{array}\right)\left(\eta_{1, k} \sigma_{1, k, j}\right)^{n-m} C_{l, j, n} \\
= & \left(\sum_{m=0}^{\infty} C_{l, j, m}\left(\eta_{1, k} \sigma_{1, k, j}\right)^{m}\right)^{-1} \sum_{n=0}^{\infty} C_{l, j, n} \sum_{m=0}^{n}\left(\begin{array}{c}
n \\
n-m
\end{array}\right)\left(\eta_{1, k} \sigma_{1, k, j}\right)^{n-m}\left(\hat{\eta}_{3, k} \hat{\sigma}_{3, k, j}\right)^{m} \\
= & \left(\sum_{m=0}^{\infty} C_{l, j, m}\left(\eta_{1, k} \sigma_{1, k, j}\right)^{m}\right)^{-1} \sum_{n=0}^{\infty} C_{l, j, n} \underbrace{\sum_{m=0}^{n}\left(\begin{array}{c}
n \\
n-m
\end{array}\right)\left(\eta_{1, k} \sigma_{1, k, j}\right)^{n-m}\left(\eta_{2, k} \sigma_{2, k, j}-\eta_{1, k} \sigma_{1, k, j}\right)^{m}}_{\left(\eta_{1, k} \sigma_{1, k, j}+\eta_{2, k} \sigma_{2, k, j}-\eta_{1, k} \sigma_{1, k, j}\right)^{n}} \\
= & \left(\sum_{m=0}^{\infty} C_{l, j, m}\left(\eta_{1, k} \sigma_{1, k, j}\right)^{m}\right)^{-1} \sum_{m=0}^{\infty} C_{l, j, m}\left(\eta_{2, k} \sigma_{2, k, j}\right)^{m}
\end{aligned}
$$

Taking logarithms on both sides yields the desired identity (82). 


\section{REFERENCES}

Abramowitz, M. and Stegun, I. A. (eds) (1964), Handbook of Mathematical Functions, National Bureau of Standards.

Airoldi, M. (2005), A Moment Expansion Approach to Option Pricing, Quantitative Finance 5, 89-104.

Amin, K. I. and Ng, V. K. (1997), Inferring Future Volatility from the Information in Implied Volatility in Eurodollar Options: A New Approach, Review of Financial Studies 10, 333-367.

Ane, T. (1999), Pricing and Hedging S\&P 500 Index Options with Hermite Polynomial Approximation: Empirical Tests of Madan and Milnes model, Journal of Futures Markets 19, 735-758.

Arrow, K. (1964), The Role of Securities in the Optimal Allocation of Risk-bearing, Review of Economic Studies 31(2), 91-96.

Backus, D., Foresi, S., Li, K. and Wu, L. (1997), Accounting for Biases in Black-Scholes, Stern School of Business, New York University, working paper .

Bahra, B. (1997), Implied Risk-Neutral Probability Density Functions from Option Prices: Theory and Application, Bank of England, working paper .

Bates, D. S. (2008), The Market for Crash Risk, Journal of Economic Dynamics and Control 32(7), 2291-2321.

Beckers, S. (1981), Standard Deviations Implied in Options Prices as Predictors of Future Stock Price Variability, Journal of Banking and Finance 5, 363-381.

Black, F. and Scholes, M. (1973), The Pricing of Options and Corporate Liabilities, Journal of Political Economy 81(3), 637-654.

Breeden, D. T. and Litzenberger, R. H. (1978), Prices of State-contingent Claims Implicit in Option Prices, Journal of Business 51(4), 621-651.

Brown, C. A. and Robinson, D. M. (2002), Skewness And Kurtosis Implied by Option Prices: A Correction, Journal of Financial Research 25(2), 279-282.

Canina, L. and Figlewski, S. (1993), The Informational Content of Implied Volatility, Review of Financial Studies 6, 659-681.

Capelle-Blancard, G., Jurczenko, E. and Maillet, B. (2001), The Approximate Option Pricing Model: Performances and Dynamic Properties, Journal of Multinational Financial Management 11, 427-443. 
Christensen, B. J. and Prabhala, N. R. (1998), The Relation Between Implied and Realized Volatility, Journal of Financial Economics 50, 125-150.

Collin-Dufresne, P. and Goldstein, R. S. (2002), Pricing Swaptions Within an Affine Framework, Journal of Derivatives.

Corrado, C. (2007), The Hidden Martingale Restriction in Gram-Charlier Option Prices, Journal of Futures Markets 27(6), 517-534.

Corrado, C. and Su, T. (1996), Skewness and Kurtosis in S\&P 500 Index Returns Implied by Option Prices, Journal of Financial Research 19(2), 175-92.

Debreu, G. (1959), Theory of Value, John Wiley \& Sons.

Flamouris, D. and Giamouridis, D. (2002), Estimating Implied PDFs from American Options on Futures: A New Semiparametric Approach, Journal of Futures Markets 22, 1-30.

Fleming, J. (1998), The Quality of Market Volatility Forecasts Implied by S\&P100 Index Options Prices, Journal of Empirical Finance 5, 317-345.

Gallant, A. R. and Nychka, D. W. (1987), Seminonparametric Maximum Likelihood Estimation, Econometrica 55, 363-390.

Geman, H., El Karoui, N. and Rochet, J.-C. (1995), Changes of Numeraire, Changes of Probability Measure and Option Pricing, Journal of Applied Probability 32, 443-458.

Harrison, J. M. and Kreps, D. M. (1979), Martingales and Arbitrage in Multiperiod Securities Markets, Journal of Economic Theory 20, 381-408.

Harrison, J. M. and Pliska, S. R. (1981), Martingales and Stochastic Integrals in the Theory of Continuous Trading, Stochastic Processes and their Applications 11, 215-260.

Harrison, J. M. and Pliska, S. R. (1983), A Stochastic Calculus Model of Continuous Trading: Complete Markets, Stochastic Processes and their Applications 15, 313-316.

Haven, E., Liu, X., Ma, C. and Shen, L. (2009), Revealing the Implied Risk-neutral MGF from Options: The Wavelet Method, Journal of Economic Dynamics and Control 33(3), 692-709.

Hida, T., Kuo, H.-H., Potthoff, J. and Streit, L. (1993), White Noise: An Infinite Dimensional Calculus, Vol. 253 of Mathematics and Its Applications, Kluwer Academic Publishers.

Jackwerth, J. C. and Rubinstein, M. (1996), Recovering Probability Distributions from Option Prices, Journal of Finance 51(5), 1611-1631. 
Jarrow, R. and Rudd, A. (1982), Approximate Option Valuation for Arbitrary Stochastic Processes, Journal of Financial Economics 10, 347-369.

Jondeau, E. and Rockinger, M. (2000), Reading the Smile: The Message Conveyed by Methods Which Infer Risk Neutral Densities, Journal of International Money and Finance 19, 885-915.

Jondeau, E. and Rockinger, M. (2001), Gram-Charlier Densities, Journal of Economic Dynamics and Control 25, 1457-1483.

Jorion, P. (1995), Predicting Volatility in the Foreign Exchange Market, Journal of Finance 50, 507-528.

Jurczenko, E., Maillet, B. and Negrea, B. (2002), Multi-moment Approximate Option Pricing Models: A General Comparison (Part 1), CNRS - University of Paris I Panthéon-Sorbonne, working paper .

Jurczenko, E., Maillet, B. and Negrea, B. (2004), A Note on Skewness and Kurtosis Adjusted Option Pricing Models under the Martingale Restriction, Quantitative Finance 4, 479-488.

Kendall, M. G. and Stuart, A. (1969), The Advanced Theory of Statistics, Vol. 1, 3 edn, Charles Griffin \& Company, London.

Knight, J. and Satchell, S. (2001), Pricing Derivatives Written on Assets with Arbitrary Skewness and Kurtosis, in J. Knight and S. Satchell (eds), Return Distributions in Finance, Butterworth and Heinemann, pp. 252-275.

Kouchard, L. (1999), Option Pricing and Higher Order Moments of the Risk-neutral Probability Density Function, University of Virginia.

Kuo, H.-H. (1996), White Noise Distribution Theory, CRC Press.

Lamoreux, C. G. and Lastrapes, W. D. (1993), Forecasting Stock-Return Variance: Toward an Understanding of Stochastic Implied Processes, Review of Financial Studies 6, 293-326.

Leon, A., Mencia, J. and Sentana, E. (2009), Parametric Properties of Semi-Nonparametric Distributions, with Applications to Option Valuation, Journal of Business \& Economic Statistics 27(2), 176192.

Margrabe, W. (1978), The Value of an Option to Exchange one Asset for Another, Journal of Finance XXXIII(1), 177-186.

Navatte, P. and Villa, C. (2000), The Information Content of Implied Volatility, Skewness and Kurtosis: Empirical Evidence from Long-term CAC 40 Options, European Financial Management 6, 41-56. 
Nikkenin, J. (2003a), Impact of Foreign Ownership Restrictions on Stock Return Distributions: Evidence from an Option Market, Journal of Multinational Financial Management 13, 141-159.

Nikkenin, J. (2003b), Normality Tests of Option-implied Risk Neutral Densities: Evidence from the Small Finnish Market, International Review of Financial Analysis 12, 99-116.

Penttinen, A. (2001), The Sensitivity of Implied Volatility to Expectations of Jumps in Volatility: An Explanation for the Illusory Bias in Implied Volatility as a Forecast, Swedish School of Economics and Business Administration, working paper .

Press, W. H., Teukolsky, S. A., Vetterling, W. T. and Flannery, B. P. (1992), Numerical Recipes in C: The Art of Scientific Computing, 2nd edn, Cambridge University Press.

Serna, G. (2004), El modelo de Corrado y Su en el mercado de opciones sobre el futuro del IBEX-35 [The Model of Corrado and Su in the Market for Options on IBEX-35 Futures], Revista de Economîa Aplicada 34, 101-125.

Skipper, M. and Buchen, P. (2003), The Quintessential Option Pricing Formula, University of Sydney, working paper .

Tanaka, K., Yamada, T. and Watanabe, T. (2010), Applications of Gram-Charlier Expansion and Bond Moments for Pricing of Interest Rates and Credit Risk, Quantitative Finance 10(6), 645-662.

Vahamaa, S. (2005), Option-implied Asymmetries in Bond Market Expectations Around Monetary Policy Actions of the ECB, Journal of Economics and Business 57, 23-38.

Vahamaa, S., Watzka, S. and Aijo, J. (2005), What Moves Options-implied Bond Market Expectations?, Journal of Futures Markets 25, 817-843.

Valle, A. L. and Calvo, G. S. (2005), Modelos alternativos de valoración de opciones sobre acciones: una aplicación al Mercado español [Alternative Valuation Models of Options on Shares: An Application to the Spanish Market], Cuadernos Económicos Información Commercial Española 69, 33-49.

Wilkens, S. (2005), Option Pricing Based on Mixtures of Distributions: Evidence from the Eurex Index and Interest Rate Futures Options Market, Derivatives Use, Trading and Regulation 11, 213-231. 